\title{
SOBOLEV-LORENTZ CAPACITY AND ITS REGULARITY IN THE EUCLIDEAN SETTING
}

\author{
Şerban Costea \\ University of Piteşti, Department of Mathematics and Computer Science \\ Str. Târgul din Vale nr. 1, RO-110040 Piteşti, Argeş, Romania; serban.costea@upit.ro
}

Dedicated to the memory of my parents Dorina and Nicolae

\begin{abstract}
This paper studies the Sobolev-Lorentz capacity and its regularity in the Euclidean setting for $n \geq 1$ integer. We extend here our previous results on the Sobolev-Lorentz capacity obtained for $n \geq 2$. Moreover, for $n \geq 2$ integer we obtain a few new results concerning the $n, 1$ relative and global capacities. Specifically, we obtain sharp estimates for the $n, 1$ relative capacity of the concentric condensers $(\bar{B}(0, r), B(0,1))$ for all $r$ in $[0,1)$. As a consequence we obtain the exact value of the $n, 1$ capacity of a point relative to all its bounded open neighborhoods from $\mathbf{R}^{n}$ when $n \geq 2$. These new sharp estimates concerning the $n, 1$ relative capacity improve some of our previous results. We also obtain a new result concerning the $n, 1$ global capacity. Namely, we show that this aforementioned constant is also the value of the $n, 1$ global capacity of any point from $\mathbf{R}^{n}$, where $n \geq 2$ is integer. Computing the aforementioned exact value of the $n, 1$ relative capacity of a point with respect to all its bounded open neighborhoods from $\mathbf{R}^{n}$ allows us to give a new prove of the embedding $H_{0}^{1,(n, 1)}(\Omega) \hookrightarrow C(\bar{\Omega}) \cap L^{\infty}(\Omega)$, where $\Omega \subset \mathbf{R}^{n}$ is open and $n \geq 2$ is an integer.

In the penultimate section of our paper we prove a new weak convergence result for bounded sequences in the non-reflexive spaces $H^{1,(p, 1)}(\Omega)$ and $H_{0}^{1,(p, 1)}(\Omega)$. The weak convergence result concerning the spaces $H^{1,(p, 1)}(\Omega)$ is valid whenever $1<p<\infty$, while the weak convergence result concerning the spaces $H_{0}^{1,(p, 1)}(\Omega)$ is valid whenever $1 \leq n<p<\infty$ or $1<n=p<\infty$. As a consequence of the weak convergence result concerning the spaces $H_{0}^{1,(p, 1)}(\Omega)$, in the last section of our paper we show that the relative and the global $(p, 1)$ and $p, 1$ capacities are Choquet whenever $1 \leq n<p<\infty$ or $1<n=p<\infty$.
\end{abstract}

\section{Introduction}

In this paper we study the Sobolev-Lorentz capacity and its regularity in the Euclidean setting for $n \geq 1$. This paper is motivated by the work of Stein-Weiss [18] and Bennett-Sharpley [1] on Lorentz spaces and by the work of Stein [17] and Cianchi-Pick [2], [3] on Sobolev-Lorentz spaces.

We studied the Sobolev-Lorentz spaces and their associated capacities extensively in our previous work. In this paper we extend some of the previous results obtained in our book [6] and in our papers [5] and [7]. In [6] we studied the Sobolev-Lorentz spaces and the associated Sobolev-Lorentz capacities in the Euclidean setting for $n \geq 2$. The restriction on $n$ there as well as in [5] was due to the fact that we studied the $n, q$ capacity for $n>1$. In our recent paper [7] we studied the Sobolev-Lorentz spaces in the Euclidean setting for $n \geq 1$. There we extended to the case $n=1$ many of the results on Sobolev-Lorentz spaces obtained in [4] and [6] for $n \geq 2$. In this paper we extend to $n=1$ many on the results on Sobolev-Lorentz capacities obtained in [4] and [6] for $n \geq 2$.

https://doi.org/10.5186/aasfm.2019.4433

2010 Mathematics Subject Classification: Primary 31C15, 46 E35.

Key words: Sobolev spaces, Lorentz spaces, capacity.

The author was partly supported by the University of Pisa via grant PRA-2015-0017. 
The Lorentz spaces were studied by Bennett-Sharpley in [1] and by Stein-Weiss in [18]. The Sobolev-Lorentz spaces have also been studied by Stein in [17], CianchiPick in [2] and [3], by Kauhanen-Koskela-Malý in [14], and by Malý-Swanson-Ziemer in [15]. We studied the Sobolev-Lorentz relative $p, q$-capacity in the Euclidean setting (see [4], [5] and [6]). See also our joint work [8] with Maz'ya.

The classical Sobolev spaces were studied by Gilbarg-Trudinger in [11], Maz'ja in [16], Evans in [10], Heinonen-Kilpeläinen-Martio in [12], and by Ziemer in [19]. The Sobolev $p$-capacity was studied by Maz'ya [16] and by Heinonen-Kilpeläinen-Martio [12] in $\mathbf{R}^{n}$.

After recalling the definition of Lorentz spaces and some of its basic properties in Section 3, we move to Section 4, where we recall the definition of the SobolevLorentz spaces and some of the results that are to be used later in the paper. In Section 5 we study the basic properties of the Sobolev-Lorentz capacities on $\mathbf{R}^{n}$ for $n \geq 1$. There we study the global Sobolev-Lorentz capacities $\operatorname{Cap}_{(p, q)}(\cdot)$ and $\operatorname{Cap}_{p, q}(\cdot)$ and the relative Sobolev-Lorentz capacities $\operatorname{cap}_{(p, q)}(\cdot, \Omega)$ and $\operatorname{cap}_{p, q}(\cdot, \Omega)$ for $\Omega \subset \mathbf{R}^{n}$ bounded and open, $n \geq 1$ integer, $1<p<\infty$ and $1 \leq q \leq \infty$. The $p$, $q$-capacity is associated to the Lorentz $p, q$-quasinorm while the $(p, q)$-capacity is associated to the Lorentz $(p, q)$-quasinorm. The case $p=q$ yields the $p$-capacity, studied extensively in literature.

In Section 5 we revisit many of the basic properties of the Sobolev-Lorentz capacities, studied extensively in Chapter 4 of our book [6] for $n \geq 2$ and we extend them to the case $n=1$. The results that we extend here concern the monotonicity, the convergence, the countable subadditivity and the regularity of these capacities. The regularity of these capacities was extended in this section to the case $n=1$ for $1<q<\infty$ when we worked with the $(p, q)$ global and the $(p, q)$ relative capacities and for $1<q<p$ when we worked with the $p, q$ global and the $p, q$ relative capacities.

Due to the non-reflexivity of the Sobolev-Lorentz spaces $H_{0}^{1,(p, 1)}(\Omega)$ and $H_{0}^{1,(p, \infty)}$ $(\Omega)$, it is challenging to prove the Choquet property for the corresponding relative and global capacities associated to these non-reflexive Sobolev-Lorentz spaces. Also, due to the fact that the $p, q$-quasinorm is not a norm when $p<q \leq \infty$, the Choquet property of the $p, q$ relative and global capacities is not known when $q$ is in the range $(p, \infty]$.

No positive results on the Choquet property for the corresponding relative and global capacities associated to these non-reflexive Sobolev-Lorentz spaces have been obtained until now. In this paper we obtain a few partial positive new results concerning the Choquet property of $(p, 1)$ and the $p, 1$ relative and global capacities. Namely, in Section 8 we show that the global Sobolev-Lorentz capacities $\operatorname{Cap}_{(p, 1)}(\cdot)$ and $\operatorname{Cap}_{p, 1}(\cdot)$ as well as the relative Sobolev-Lorentz capacities $\operatorname{cap}_{(p, 1)}(\cdot, \Omega)$ and $\operatorname{cap}_{p, 1}(\cdot, \Omega)$ are Choquet whenever $1 \leq n<p<\infty$ or $1<n=p<\infty$. Here $\Omega \subset \mathbf{R}^{n}$ is a bounded and open set and $n \geq 1$ is an integer. See Theorems 8.2 and 8.3 for the regularity of the relative capacities. Theorems 8.5 and 8.6 deal with the regularity of the global capacities.

In order to prove the regularity of these capacities we needed to prove a Monotone Convergence Theorem for each of them. See Theorem 8.1 for the relative capacities and Theorem 8.4 for the global capacities. These are new results as well. When proving the Choquet property of the $(p, 1)$ and $p, 1$ relative and global capacities for these values of $n$ and $p$ (that is, $1 \leq n<p<\infty$ or $1<n=p<\infty$ ) we used many times the fact that for these values of $n$ and $p$ we can work with continuous admissible functions from $H_{0}^{1,(p, 1)}(\Omega)$. 
Indeed, in [7] we proved that the spaces $H_{0}^{1,(p, q)}(\Omega)$ embed into the space $C^{1-\frac{n}{p}}(\bar{\Omega})$ of Hölder continuous functions on $\bar{\Omega}$ with exponent $1-\frac{n}{p}$ whenever $\Omega \subset \mathbf{R}^{n}$ is open and $1 \leq n, q \leq \infty$. See [7, Theorem 5.5 (iii)] for $1=n<p<\infty$ and [7, Theorem 5.6 (iv)] for $1<n<p<\infty$. For $1<n=p<\infty$ we give a new prove of the embedding $H_{0}^{1,(n, 1)}(\Omega) \hookrightarrow C(\bar{\Omega}) \cap L^{\infty}(\Omega)$ in section 6 and we find the optimal constant for the embedding. See Theorem 6.4 (ii). This embedding was proved by Stein in his paper [17] and by Cianchi-Pick (see [2, Theorem 3.5 (i)]) with the same optimal constant that we obtained in this paper.

Our proof of this embedding is different. We use a new approach. Specifically, we use the theory of the $n, 1$ relative capacity in $\mathbf{R}^{n}, n \geq 2$. In Section 6 we obtain a new result by improving our estimates from [5, Theorem 3.11] for the $n, 1$ relative capacity of the condensers $(\bar{B}(0, r), B(0,1))$ and extending them to ALL $r$ in $[0,1)$. See Theorem 6.2 (i). In particular, we obtain the exact value for the $n, 1$ capacity of a point relative to all its bounded open neighborhoods from $\mathbf{R}^{n}$, a strictly positive number as we saw in [5, Corollary 3.8]. See Theorem 6.2 (ii). Moreover, in this section we obtain a new result for the global $n, 1$ capacity as well. Namely, in Theorem 6.3 we show that the value from Theorem 6.2 (ii) is also the value of the global $n, 1$ capacity of any point from $\mathbf{R}^{n}$.

By using the theory of the $n, 1$ relative capacity in $\mathbf{R}^{n}, n \geq 2$, we see that this aforementioned constant shows up in the embedding $H_{0}^{1,(n, 1)}(\Omega) \hookrightarrow C(\bar{\Omega}) \cap L^{\infty}(\Omega)$. See (3). Thus, Section 6 together with our paper [7] (see [7, Theorems 3.5, 4.3, 4.13 and 5.6]) reinforce the fact that for every $n \geq 2$ integer and for every $\Omega \subset \mathbf{R}^{n}$ open, the space $H_{\text {loc }}^{1,(n, 1)}(\Omega)$ is the largest Sobolev-Lorentz space defined on $\Omega$ for which each function has a version in $C(\Omega)$.

This embedding result from Section 6 is being relied on heavily in Section 7 and in Section 8.

In Section 7 we prove a new weak convergence result for bounded sequences in the non-reflexive spaces $H^{1,(p, 1)}(\Omega)$ and $H_{0}^{1,(p, 1)}(\Omega)$. This convergence result applied to bounded sequences from $H^{1,(p, 1)}(\Omega)$ (or from $H_{0}^{1,(p, 1)}(\Omega)$ ) appears to yield less than the existence of subsequences that converge weakly in $L^{p, 1}(\Omega) \times L^{p, 1}\left(\Omega ; \mathbf{R}^{n}\right)$ in the classical sense. See Theorem 7.1. This new weak convergence result concerning $H^{1,(p, 1)}(\Omega)$ holds for all $p$ in $(1, \infty)$ and for all integers $n \geq 1$. See Theorem 7.1 (i). We fix $q$ in $(1, \infty)$. We show that even in a non-reflexive space such as $H^{1,(p, 1)}(\Omega)$, if we have a bounded sequence $u_{k}$ in $H^{1,(p, 1)}(\Omega)$ such that $\left(u_{k}, \nabla u_{k}\right)$ converges weakly to $(u, \nabla u)$ in $L^{(p, q)}(\Omega) \times L^{(p, q)}\left(\Omega ; \mathbf{R}^{n}\right)$, then the function $u$ is in the reflexive space $H^{1,(p, s)}(\Omega)$ and in fact $\left(u_{k}, \nabla u_{k}\right)$ converges weakly to $(u, \nabla u)$ in $L^{(p, s)}(\Omega) \times L^{(p, s)}\left(\Omega ; \mathbf{R}^{n}\right)$ whenever $1<s<\infty$.

Then we show that this limit function $u$ is also in the non-reflexive space $H^{1,(p, 1)}(\Omega)$. This task is challenging to prove. Due to the non-reflexivity of the spaces $L^{p, 1}\left(\Omega ; \mathbf{R}^{m}\right)$, we do not know whether the sequence $\left(u_{k}, \nabla u_{k}\right)$ converges weakly to $(u, \nabla u)$ in $L^{p, 1}(\Omega) \times L^{p, 1}\left(\Omega ; \mathbf{R}^{n}\right)$ or not. Although we cannot rely on the weak- ${ }^{\star}$ lower semicontinuity of the $p, 1$ norm, we manage to prove the membership of $u$ in $H^{1,(p, 1)}(\Omega)$ and a Fatou-type result for $u$ and for $\nabla u$ with respect to both the $p, 1$ norm and the $(p, 1)$ norm.

The new weak convergence result for $H_{0}^{1,(p, 1)}(\Omega)$ is even more challenging to prove. We managed to prove it for $1 \leq n<p<\infty$ and for $1<n=p<\infty$. See Theorem 7.1 (ii). When proving this weak convergence result for $H_{0}^{1,(p, 1)}(\Omega)$ we relied heavily many times on the fact that for these values of $n$ and $p$ we can work with 
continuous functions from $H_{0}^{1,(p, 1)}(\Omega)$. This leaves for instance as an open question the membership of the limit function $u$ in $H_{0}^{1,(p, 1)}(\Omega)$ when $1<p<n, \Omega \subset \mathbf{R}^{n}$ is bounded and $u$ is not compactly supported in $\Omega$.

This new weak convergence theorem from Section 7 is being put to use later in Section 8 to prove the Choquet property of the global Sobolev-Lorentz capacities $\operatorname{Cap}_{(p, 1)}(\cdot)$ and $\operatorname{Cap}_{p, 1}(\cdot)$ and of the relative Sobolev-Lorentz capacities $\operatorname{cap}_{(p, 1)}(\cdot, \Omega)$ and $\operatorname{cap}_{p, 1}(\cdot, \Omega)$ whenever $1 \leq n<p<\infty$ or $1<n=p<\infty$. Like before, $\Omega \subset \mathbf{R}^{n}$ is a bounded and open set and $n \geq 1$ is an integer. The existence of discontinuous and/or unbounded functions in $H_{0}^{1,(p, 1)}(\Omega)$ when $1<p<n$ prevents us for now from extending the Choquet property of the $p, 1$ and $(p, 1)$ relative and global capacities to the case $1<p<n$.

\section{Notations}

Here we recall the standard notation to be used throughout this paper. (See also [7]). Throughout this paper, $C$ will denote a positive constant whose value is not necessarily the same at each occurrence; it may vary even within a line. $C(a, b, \ldots)$ is a constant that depends only on the parameters $a, b, \cdots$.

Throughout this paper $\Omega$ will denote a nonempty open subset of $\mathbf{R}^{n}$, while $d x=$ $d m_{n}(x)$ will denote the Lebesgue $n$-measure in $\mathbf{R}^{n}$, where $n \geq 1$ is an integer. For $E \subset \mathbf{R}^{n}$, the boundary, the closure, and the complement of $E$ with respect to $\mathbf{R}^{n}$ will be denoted by $\partial E, \bar{E}$, and $\mathbf{R}^{n} \backslash E$, respectively, while $|E|=\int_{E} d x$ will denote the Lebesgue measure of $E$ whenever $E$ is measurable; $E \subset \subset F$ means that $\bar{E}$ is a compact subset of $F$. Moreover, $B(a, r)=\left\{x \in \mathbf{R}^{n}:|x-a|<r\right\}$ is the open ball with center $a \in \mathbf{R}^{n}$ and radius $r>0$, while $\bar{B}(a, r)=\left\{x \in \mathbf{R}^{n}:|x-a| \leq r\right\}$ is the closed ball with center $a \in \mathbf{R}^{n}$ and radius $r>0$.

For $n \geq 1$ integer, $\Omega_{n}$ denotes the Lebesgue measure of the $n$-dimensional unit ball. (That is, $\Omega_{n}=|B(0,1)|$ ). For $n \geq 2$ integer, $\omega_{n-1}$ denotes the spherical measure of the $n$-1-dimensional sphere; thus, $\omega_{n-1}=n \Omega_{n}$ for every integer $n \geq 2$. For a Lebesgue measurable function $u: \Omega \rightarrow \mathbf{R}$, supp $u$ is the smallest closed set such that $u$ vanishes outside supp $u$. For a Lebesgue measurable vector-valued function $f=\left(f_{1}, \ldots, f_{m}\right): \Omega \rightarrow \mathbf{R}^{m}$, we let

$$
|f|=\sqrt{f_{1}^{2}+f_{2}^{2}+\ldots+f_{m}^{2}}
$$

\section{Lorentz spaces}

For the next three subsections we follow mostly our paper [7].

3.1. Definitions and basic properties. Let $f: \Omega \rightarrow \mathbf{R}$ be a measurable function. We define $\lambda_{[f]}$, the distribution function of $f$ as follows (see BennettSharpley [1, Definition II.1.1] and Stein-Weiss [18, p. 57]):

$$
\lambda_{[f]}(t)=|\{x \in \Omega:|f(x)|>t\}|, \quad t \geq 0 .
$$

We define $f^{*}$, the nonincreasing rearrangement of $f$ by

$$
f^{*}(t)=\inf \left\{v: \lambda_{[f]}(v) \leq t\right\}, \quad t \geq 0 .
$$

(See Bennett-Sharpley [1, Definition II.1.5] and Stein-Weiss [18, p. 189]). We notice that $f$ and $f^{*}$ have the same distribution function. Moreover, for every positive $\alpha$ we 
have $\left(|f|^{\alpha}\right)^{*}=\left(|f|^{*}\right)^{\alpha}$ and if $|g| \leq|f|$ a.e. on $\Omega$, then $g^{*} \leq f^{*}$. (See Bennett-Sharpley [1, Proposition II.1.7]). We also define $f^{* *}$, the maximal function of $f^{*}$ by

$$
f^{* *}(t)=m_{f^{*}}(t)=\frac{1}{t} \int_{0}^{t} f^{*}(s) d s, \quad t>0 .
$$

(See Bennett-Sharpley [1, Definition II.3.1] and Stein-Weiss [18, p. 203]).

Throughout this paper, we denote by $q^{\prime}$ the Hölder conjugate of $q \in[1, \infty]$. The Lorentz space $L^{p, q}(\Omega), 1<p<\infty, 1 \leq q \leq \infty$, is defined as follows:

$$
L^{p, q}(\Omega)=\left\{f: \Omega \rightarrow \mathbf{R}: f \text { is measurable and }\|f\|_{L^{p, q}(\Omega)}<\infty\right\},
$$

where

$$
\|f\|_{L^{p, q}(\Omega)}=\|f\|_{p, q}= \begin{cases}\left(\int_{0}^{\infty}\left(t^{\frac{1}{p}} f^{*}(t)\right)^{q} \frac{d t}{t}\right)^{\frac{1}{q}}, & 1 \leq q<\infty, \\ \sup _{t>0} t \lambda_{[f]}(t)^{\frac{1}{p}}=\sup _{s>0} s^{\frac{1}{p}} f^{*}(s), & q=\infty .\end{cases}
$$

(See Bennett-Sharpley [1, Definition IV.4.1] and Stein-Weiss [18, p. 191]). If $1 \leq$ $q \leq p$, then $\|\cdot\|_{L^{p, q}(\Omega)}$ already represents a norm (see Bennett-Sharpley [1, Theorem IV.4.3]); when $1<p<q \leq \infty,\|\cdot\|_{L^{p, q}(\Omega)}$ represents a quasinorm that is equivalent to the norm $\|\cdot\|_{L^{(p, q)}(\Omega)}$, where

$$
\|f\|_{L^{(p, q)}(\Omega)}=\|f\|_{(p, q)}= \begin{cases}\left(\int_{0}^{\infty}\left(t^{\frac{1}{p}} f^{* *}(t)\right)^{q} \frac{d t}{t}\right)^{\frac{1}{q}}, & 1 \leq q<\infty, \\ \sup _{t>0} t^{\frac{1}{p}} f^{* *}(t), & q=\infty .\end{cases}
$$

(See Bennett-Sharpley [1, Definition IV.4.4]). Namely, from Lemma IV.4.5 in BennettSharpley [1] we have that

$$
\|f\|_{L^{p, q}(\Omega)} \leq\|f\|_{L^{(p, q)}(\Omega)} \leq \frac{p}{p-1}\|f\|_{L^{p, q}(\Omega)}
$$

for every $1 \leq q \leq \infty$.

For a measurable vector-valued function $f=\left(f_{1}, \ldots, f_{m}\right): \Omega \rightarrow \mathbf{R}^{m}$ we say that $f \in L^{p, q}\left(\Omega ; \mathbf{R}^{m}\right)$ if and only if $f_{i} \in L^{p, q}(\Omega)$ for $i=1,2, \ldots, m$, if and only if $|f| \in L^{p, q}(\Omega)$ and we define

$$
\|f\|_{L^{p, q}\left(\Omega ; \mathbf{R}^{m}\right)}=\||f|\|_{L^{p, q}(\Omega)} .
$$

Similarly

$$
\|f\|_{L^{(p, q)}\left(\Omega ; \mathbf{R}^{m}\right)}=\||f|\|_{L^{(p, q)}(\Omega)} .
$$

Obviously, it follows from the real-valued case that

$$
\|f\|_{L^{p, q}\left(\Omega ; \mathbf{R}^{m}\right)} \leq\|f\|_{L^{(p, q)}\left(\Omega ; \mathbf{R}^{m}\right)} \leq \frac{p}{p-1}\|f\|_{L^{p, q}\left(\Omega ; \mathbf{R}^{m}\right)}
$$

for every $1 \leq q \leq \infty$, and like in the real-valued case, $\|\cdot\|_{L^{p, q}\left(\Omega ; \mathbf{R}^{m}\right)}$ is already a norm when $1 \leq q \leq p$, while it is a quasinorm when $1<p<q \leq \infty$.

It is known that $\left(L^{p, q}\left(\Omega ; \mathbf{R}^{m}\right),\|\cdot\|_{L^{p, q}\left(\Omega ; \mathbf{R}^{m}\right)}\right)$ is a Banach space for $1 \leq q \leq p$, while $\left(L^{p, q}\left(\Omega ; \mathbf{R}^{m}\right),\|\cdot\|_{L^{(p, q)}\left(\Omega ; \mathbf{R}^{m}\right)}\right)$ is a Banach space for $1<p<\infty, 1 \leq q \leq \infty$. For more results on Lorentz spaces we refer the reader to Bennett-Sharpley [1, Chapter IV] and to Stein-Weiss [18, Chapter V]. 


\subsection{Weak convergence of the $(p, q)$-norm and reflexivity of the Lorentz spaces.}

Definition 3.1. Let $\Omega$ be an open set in $\mathbf{R}^{n}$, where $n \geq 1$ is an integer. Suppose $1<p<\infty$ and $1 \leq q<\infty$. We say that a sequence $u_{j}$ in $L^{(p, q)}(\Omega)$ converges weakly to a function $u \in L^{(p, q)}(\Omega)$ if

$$
\int_{\Omega} v(x) u_{j}(x) d x \rightarrow \int_{\Omega} v(x) u(x) d x
$$

whenever $v \in L^{\left(p^{\prime}, q^{\prime}\right)}(\Omega)$. There is an obvious interpretation in terms of the coordinate functions for the weak convergence of vector-valued functions in $L^{(p, q)}\left(\Omega ; \mathbf{R}^{m}\right)$, where $m \geq 1$ is an integer.

The spaces $L^{p, q}\left(\Omega ; \mathbf{R}^{m}\right)$ are reflexive whenever $1<q<\infty$ and the dual of $L^{p, q}\left(\Omega ; \mathbf{R}^{m}\right)$ is, up to equivalence of norms, the space $L^{p^{\prime}, q^{\prime}}\left(\Omega ; \mathbf{R}^{m}\right)$ for $1 \leq q<\infty$. See Bennett-Sharpley [1, Theorem IV.4.7 and Corollary IV.4.8], Hunt [13, p. 259$262]$ and the definition of the spaces $L^{p, q}\left(\Omega ; \mathbf{R}^{m}\right)$. We notice that the terminology in the previous definition agrees with the usual weak convergence in the Banach space theory if $1 \leq q<\infty$.

\subsection{Strict inclusions between Lorentz spaces.}

Remark 3.2. It is known (see Bennett-Sharpley [1, Proposition IV.4.2]) that for every $p \in(1, \infty)$ and $1 \leq r<s \leq \infty$ there exists a constant $C(p, r, s)>0$ such that

$$
\|f\|_{L^{p, s}(\Omega)} \leq C(p, r, s)\|f\|_{L^{p, r}(\Omega)}
$$

for all measurable functions $f \in L^{p, r}(\Omega)$. In particular, $L^{p, r}(\Omega) \subset L^{p, s}(\Omega)$. Like in the real-valued case, it follows that

$$
\|f\|_{L^{p, s}\left(\Omega ; \mathbf{R}^{m}\right)} \leq C(p, r, s)\|f\|_{L^{p, r}\left(\Omega ; \mathbf{R}^{m}\right)}
$$

for every $m \geq 1$ integer and for all measurable functions $f \in L^{p, r}\left(\Omega ; \mathbf{R}^{m}\right)$, where $C(p, r, s)$ is the constant from (1). In particular,

$$
L^{p, r}\left(\Omega ; \mathbf{R}^{m}\right) \subset L^{p, s}\left(\Omega ; \mathbf{R}^{m}\right) \text { for every } m \geq 1 \text { integer. }
$$

The above inclusion is strict. See Ziemer [19, p. 37, Exercise 1.7] and [7, Theorems 3.4 and 3.5].

\section{Sobolev-Lorentz Spaces}

This section is based in part on Chapter 3 of our book [6] and on Section 4 of our article [7].

4.1. The $H^{1,(p, q)}$ and $W^{1,(p, q)}$ spaces. In this subsection we recall the definition of the Sobolev-Lorentz spaces $H_{0}^{1,(p, q)}(\Omega), H^{1,(p, q)}(\Omega)$, and $W^{1,(p, q)}(\Omega)$, where $\Omega \subset \mathbf{R}^{n}$ is an open set and $n \geq 1$ is an integer. These spaces were studied extensively in Chapter 3 of our book [6] (the case $n \geq 2$ ) and in Section 4 of our article [7] (the case $n \geq 1$ ).

For $1<p<\infty$ and $1 \leq q \leq \infty$ we define the Sobolev-Lorentz space $H^{1,(p, q)}(\Omega)$ as follows. Let $r=\min (p, q)$. For a function $\phi \in C^{\infty}(\Omega)$ we define its Sobolev-Lorentz $(p, q)$-norm by

$$
\|\phi\|_{1,(p, q) ; \Omega}=\left(\|\phi\|_{L^{(p, q)}(\Omega)}^{r}+\|\nabla \phi\|_{L^{(p, q)}\left(\Omega ; \mathbf{R}^{n}\right)}^{r}\right)^{1 / r}
$$


where $\nabla \phi=\left(\partial_{1} \phi, \ldots, \partial_{n} \phi\right)$ is the gradient of $\phi$. Similarly we define the SobolevLorentz $p, q$-quasinorm of $\phi$ by

$$
\|\phi\|_{1, p, q ; \Omega}=\left(\|\phi\|_{L^{p, q}(\Omega)}^{r}+\|\nabla \phi\|_{L^{p, q}\left(\Omega ; \mathbf{R}^{n}\right)}^{r}\right)^{1 / r},
$$

Then $H^{1,(p, q)}(\Omega)$ is defined as the completion of

$$
\left\{\phi \in C^{\infty}(\Omega):\|\phi\|_{1,(p, q) ; \Omega}<\infty\right\}
$$

with respect to the norm $\|\cdot\|_{1,(p, q) ; \Omega}$. Throughout the paper we use $\|\cdot\|_{H^{1,(p, q)}(\Omega)}$ instead of $\|\cdot\|_{1,(p, q) ; \Omega}$ and $\|\cdot\|_{H^{1, p, q}(\Omega)}$ instead of $\|\cdot\|_{1, p, q ; \Omega}$.

The Sobolev-Lorentz space $H_{0}^{1,(p, q)}(\Omega)$ is defined as the closure of $C_{0}^{\infty}(\Omega)$ in $H^{1,(p, q)}(\Omega)$. From the discussion in subsection 4.1 of our paper [7], we have that the Sobolev-Lorentz spaces $H_{0}^{1,(p, q)}(\Omega)$ and $H^{1,(p, q)}(\Omega)$ are reflexive Banach spaces when $1<q<\infty$. From the same discussion it follows that $H_{0}^{1,(p, 1)}(\Omega)$ and $H^{1,(p, 1)}(\Omega)$ are non-reflexive Banach spaces.

Let $u \in L_{\text {loc }}^{1}(\Omega)$. For $i=1, \ldots, n$ a function $v \in L_{\text {loc }}^{1}(\Omega)$ is called the $i$ th weak partial derivative of $u$ and we denote $v=\partial_{i} u$ if

$$
\int_{\Omega} \varphi(x) v(x) d x=-\int_{\Omega} \partial_{i} \varphi(x) u(x) d x
$$

for all $\varphi \in C_{0}^{\infty}(\Omega)$. We define the Sobolev-Lorentz space $W^{1,(p, q)}(\Omega)$ by

$$
W^{1,(p, q)}(\Omega)=L^{(p, q)}(\Omega) \cap\left\{u: \partial_{i} u \in L^{(p, q)}(\Omega), i=1, \ldots, n\right\} .
$$

The space $W^{1,(p, q)}(\Omega)$ is equipped with the norm

$$
\|u\|_{W^{1,(p, q)}(\Omega)}=\|u\|_{L^{(p, q)}(\Omega)}+\sum_{i=1}^{n}\left\|\partial_{i} u\right\|_{L^{(p, q)}(\Omega)},
$$

which is clearly equivalent to

$$
\left(\|u\|_{L^{(p, q)}(\Omega)}^{r}+\|\nabla u\|_{L^{(p, q)}\left(\Omega ; \mathbf{R}^{n}\right)}^{r}\right)^{1 / r}
$$

where $r=\min (p, q)$. Here $\nabla u$ is the distributional gradient of $u$.

In [7, Theorem 4.8] we showed that $H^{1,(p, \infty)}(\Omega) \subsetneq W^{1,(p, \infty)}(\Omega)$ and that the spaces $H_{0}^{1,(p, \infty)}(\Omega), H^{1,(p, \infty)}(\Omega)$ and $W^{1,(p, \infty)}(\Omega)$ are not reflexive. Furthermore, in [7, Theorem 4.11] we proved that $H^{1,(p, q)}(\Omega)=W^{1,(p, q)}(\Omega)$ whenever $1 \leq q<\infty$. The corresponding local space $H_{\mathrm{loc}}^{1,(p, q)}(\Omega)$ is defined in the obvious manner: $u$ is in $H_{\mathrm{loc}}^{1,(p, q)}(\Omega)$ if and only if $u$ is in $H^{1,(p, q)}\left(\Omega^{\prime}\right)$ for every open set $\Omega^{\prime} \subset \subset \Omega$. Similarly, the local space $W_{\text {loc }}^{1,(p, q)}(\Omega)$ is defined as follows: $u$ is in $W_{\text {loc }}^{1,(p, q)}(\Omega)$ if and only if $u$ is in $W^{1,(p, q)}\left(\Omega^{\prime}\right)$ for every open set $\Omega^{\prime} \subset \subset \Omega$.

For more details on these spaces including their basic properties we refer the readers to Chapter 3 of our book [6] and to Section 4 of our article [7].

4.2. Product rule. Next we record the following lemma which says that the product between a function $u$ in $H^{1,(p, q)}(\Omega)$ and a function $\varphi$ in $C_{0}^{\infty}(\Omega)$ yields a function in $H_{0}^{1,(p, q)}(\Omega)$ if $1<p<\infty$ and $1 \leq q \leq \infty$. See also [7, Lemma 4.9 and Theorem 4.11]).

Lemma 4.1. Let $\Omega \subset \mathbf{R}^{n}$ be an open set, where $n \geq 1$ is an integer. Suppose that $1<p<\infty$ and $1 \leq q \leq \infty$. Suppose that $u \in H^{1,(p, q)}(\Omega)$ and that $\varphi \in C_{0}^{\infty}(\Omega)$. Then $u \varphi \in H_{0}^{1,(p, q)}(\Omega)$ and $\nabla(u \varphi)=u \nabla \varphi+\varphi \nabla u$. 
Remark 4.2. Lemma 4.1 easily implies

$$
\begin{aligned}
\|u \varphi\|_{W^{1,(p, q)}(\Omega)} \leq & \|\varphi\|_{L^{\infty}(\Omega)}\|u\|_{L^{(p, q)}(\Omega)}+\|\varphi\|_{L^{\infty}(\Omega)}\left(\sum_{i=1}^{n}\left\|\partial_{i} u\right\|_{L^{(p, q)}(\Omega)}\right) \\
& +\left(\sum_{i=1}^{n}\left\|\partial_{i} \varphi\right\|_{L^{\infty}(\Omega)}\right)\|u\|_{L^{(p, q)}(\Omega)} \\
\leq & \left(\|\varphi\|_{L^{\infty}(\Omega)}+\sum_{i=1}^{n}\left\|\partial_{i} \varphi\right\|_{L^{\infty}(\Omega)}\right)\|u\|_{W^{1,(p, q)}(\Omega)}
\end{aligned}
$$

for every $u \in H^{1,(p, q)}(\Omega)$ and

$$
\begin{aligned}
\|u \varphi\|_{H^{1,(p, q)}(\Omega)} & =\left(\|u \varphi\|_{L^{(p, q)}(\Omega)}^{r}+\|\nabla(u \varphi)\|_{L^{(p, q)}\left(\Omega ; \mathbf{R}^{n}\right)}^{r}\right)^{1 / r} \\
& =\left(\|u \varphi\|_{L^{(p, q)}(\Omega)}^{r}+\|\varphi \nabla u+u \nabla \varphi\|_{L^{(p, q)}\left(\Omega ; \mathbf{R}^{n}\right)}^{r}\right)^{1 / r} \\
& \leq\left(\|u \varphi\|_{L^{(p, q)}(\Omega)}^{r}+\|\varphi \nabla u\|_{L^{(p, q)}\left(\Omega ; \mathbf{R}^{n}\right)}^{r}\right)^{1 / r}+\|u \nabla \varphi\|_{L^{(p, q)}\left(\Omega ; \mathbf{R}^{n}\right)} \\
& \leq\|\varphi\|_{L^{\infty}(\Omega)}\|u\|_{H^{1,(p, q)}(\Omega)}+\|\nabla \varphi\|_{L^{\infty}(\Omega)}\|u\|_{L^{(p, q)}\left(\Omega ; \mathbf{R}^{n}\right)} \\
& \leq\left(\|\varphi\|_{L^{\infty}(\Omega)}+\|\nabla \varphi\|_{L^{\infty}(\Omega)}\right)\|u\|_{H^{1,(p, q)}(\Omega)}
\end{aligned}
$$

for every $u \in H^{1,(p, q)}(\Omega)$; here $1 \leq q \leq \infty$ and $r=\min (p, q)$, like in the definition of the $\|\cdot\|_{H^{1,(p, q)}(\Omega)}$ norm.

4.3. Reflexivity results. Next we recall the following reflexivity results from [6] concerning the Sobolev-Lorentz spaces, valid for all integers $n \geq 1$ and for all $q$ in $(1, \infty)$. Both these results are standard applications of Mazur's lemma.

Theorem 4.3. (See [4, Theorem V.20] and [6, Theorem 3.5.2]) Let $1<p, q<$ $\infty$. Suppose that $\mathcal{K}$ is a convex and closed set of $H^{1,(p, q)}(\Omega)$. If $u_{j} \in \mathcal{K}$ is a sequence and if $u \in L^{(p, q)}(\Omega)$ and $v \in L^{(p, q)}\left(\Omega ; \mathbf{R}^{n}\right)$ are functions such that $u_{j} \rightarrow u$ weakly in $L^{p, q}(\Omega)$ and $\nabla u_{j} \rightarrow v$ weakly in $L^{p, q}\left(\Omega ; \mathbf{R}^{n}\right)$, then $u \in \mathcal{K}$ and $v=\nabla u$.

Theorem 4.4. (See [4, Theorem V.21] and [6, Theorem 3.5.3]) Let $1<p, q<$ $\infty$. Suppose that $u_{j}$ is a bounded sequence in $H^{1,(p, q)}(\Omega)$. Then there is a subsequence $u_{j_{i}}$ and a function $u \in H^{1,(p, q)}(\Omega)$ such that $u_{j_{i}} \rightarrow u$ weakly in $L^{p, q}(\Omega)$ and $\nabla u_{j_{i}} \rightarrow \nabla u$ weakly in $L^{p, q}\left(\Omega ; \mathbf{R}^{n}\right)$. Moreover, if $u_{j} \in H_{0}^{1,(p, q)}(\Omega)$ for all $j \geq 1$, then $u \in H_{0}^{1,(p, q)}(\Omega)$.

\section{Sobolev-Lorentz Capacity}

This section is based on Chapter 4 of our book [6]. In [6] we studied the SobolevLorentz relative and global capacities for $1<p<\infty, 1 \leq q \leq \infty$ and $n>1$ integer. There we developed a capacity theory based on the definition of Sobolev functions on $\mathbf{R}^{n}$ with respect to the Lorentz norm. Basic properties of capacity, including monotonicity, countable subadditivity and several convergence results were included there. All those results were proved in [6] for $n \geq 2$ but they can be extended to the case $n=1$. We do it here, in this section of our paper.

5.1. The Sobolev-Lorentz $(\boldsymbol{p}, \boldsymbol{q})$ relative capacity. Let $n \geq 1$ be an integer. Suppose $1<p<\infty$ and $1 \leq q \leq \infty$. Let $\Omega \subset \mathbf{R}^{n}$ be a bounded open set and let $E$ be a subset of $\Omega$. The Sobolev-Lorentz $(p, q)$ relative capacity of the pair $(E, \Omega)$ is 
denoted

$$
\operatorname{cap}_{(p, q)}(E, \Omega)=\inf \left\{\|\nabla u\|_{L^{(p, q)}\left(\Omega ; \mathbf{R}^{n}\right)}^{p}: u \in \mathcal{A}(E, \Omega)\right\}
$$

where

$$
\mathcal{A}(E, \Omega)=\left\{u \in H_{0}^{1,(p, q)}(\Omega): u \geq 1 \text { in a neighborhood of } E\right\} .
$$

We call $\mathcal{A}(E, \Omega)$ the set of admissible functions for the condenser $(E, \Omega)$. If $\mathcal{A}(E, \Omega)=$ $\emptyset$, we set $\operatorname{cap}_{(p, q)}(E, \Omega)=\infty$.

Since $H_{0}^{1,(p, q)}(\Omega)$ is closed under truncations from below by 0 and from above by 1 and since these truncations do not increase the $(p, q)$-norm of the distributional gradients, it is enough to consider only those admissible functions $u$ for which $0 \leq$ $u \leq 1$.

5.1.1. Basic properties of the $(p, q)$ relative capacity. Usually, a capacity is a monotone and subadditive set function. The following theorem will show, among other things, that this is true in the case of the $(p, q)$ relative capacity. In our thesis [4] we studied only the case $1<n=p<\infty$. In [6] we extended the results from [4] to the case $1<p<\infty$ and $n>1$. The following theorem generalizes Theorem V.23 from [4] and Theorem 4.1.1 from [6] to the case $1<p<\infty$ and $n=1$.

Theorem 5.1. (See [4, Theorem V.23] and [6, Theorem 4.1.1]) Let $n \geq 1$ be an integer. Suppose $1<p<\infty$ and $1 \leq q \leq \infty$. Let $\Omega \subset \mathbf{R}^{n}$ be a bounded open set. The set function $E \mapsto \operatorname{cap}_{(p, q)}(E, \Omega), E \subset \Omega$, enjoys the following properties:

(i) If $E_{1} \subset E_{2}$, then $\operatorname{cap}_{(p, q)}\left(E_{1}, \Omega\right) \leq \operatorname{cap}_{(p, q)}\left(E_{2}, \Omega\right)$.

(ii) If $\Omega_{1} \subset \Omega_{2}$ are open and bounded and $E \subset \Omega_{1}$, then

$$
\operatorname{cap}_{(p, q)}\left(E, \Omega_{2}\right) \leq \operatorname{cap}_{(p, q)}\left(E, \Omega_{1}\right) .
$$

(iii) $\operatorname{cap}_{(p, q)}(E, \Omega)=\inf \left\{\operatorname{cap}_{(p, q)}(U, \Omega): E \subset U \subset \Omega, U\right.$ open $\}$.

(iv) If $K_{i}$ is a decreasing sequence of compact subsets of $\Omega$ with $K=\bigcap_{i=1}^{\infty} K_{i}$, then

$$
\operatorname{cap}_{(p, q)}(K, \Omega)=\lim _{i \rightarrow \infty} \operatorname{cap}_{(p, q)}\left(K_{i}, \Omega\right) .
$$

(v) Suppose that $1<q<\infty$. If $E_{1} \subset E_{2} \subset \ldots \subset E=\bigcup_{i=1}^{\infty} E_{i} \subset \Omega$, then

$$
\operatorname{cap}_{(p, q)}(E, \Omega)=\lim _{i \rightarrow \infty} \operatorname{cap}_{(p, q)}\left(E_{i}, \Omega\right) .
$$

(vi) If $E=\bigcup_{i=1}^{\infty} E_{i} \subset \Omega$, then

$$
\operatorname{cap}_{(p, q)}(E, \Omega)^{1 / p} \leq \sum_{i=1}^{\infty} \operatorname{cap}_{(p, q)}\left(E_{i}, \Omega\right)^{1 / p} .
$$

Proof. This result was proved in [6] for $n \geq 2$. See [6, Theorem 4.1.1]. The proof of the case $n=1$ is very similar to the proof of [6, Theorem 4.1.1] and omitted.

The set function $\operatorname{cap}_{(p, q)}(\cdot, \Omega)$ satisfies properties (i), (iv), and (v) of Theorem 5.1 whenever $1<p, q<\infty$ and $\Omega$ is a bounded open set in $\mathbf{R}^{n}$, where $n \geq 1$ is an integer. Thus, $\operatorname{cap}_{(p, q)}(\cdot, \Omega)$ is a Choquet capacity (relative to $\Omega$ ) whenever $1<p, q<\infty$ and $\Omega$ is a bounded open set in $\mathbf{R}^{n}$, where $n \geq 1$ is an integer. We may thus invoke an important capacitability theorem of Choquet and state the following result. See Doob [9, Appendix II].

Theorem 5.2. Let $\Omega$ be a bounded open set in $\mathbf{R}^{n}$, where $n \geq 1$ is an integer. Suppose $1<p, q<\infty$. The set function $E \mapsto \operatorname{cap}_{(p, q)}(E, \Omega), E \subset \Omega$, is a Choquet 
capacity. In particular, all Borel subsets (in fact, all analytic) subsets $E$ of $\Omega$ are capacitable, i.e.

$$
\operatorname{cap}_{(p, q)}(E, \Omega)=\sup \left\{\operatorname{cap}_{(p, q)}(K, \Omega): K \subset E \text { compact }\right\}
$$

The set function $\operatorname{cap}_{(p, q)}(\cdot, \Omega)$ satisfies properties (i) and (iv) of Theorem 5.1 whenever $q=1$ or $q=\infty$. Like in Theorem 5.1 , the set $\Omega$ is bounded and open in $\mathbf{R}^{n}$, where $n \geq 1$ is an integer.

Question 5.3. Let $\Omega$ be a bounded open set in $\mathbf{R}^{n}$, where $n \geq 1$ is an integer. Suppose $1<p<\infty$. Is $\operatorname{cap}_{(p, q)}(\cdot, \Omega)$ a Choquet capacity when $q=1$ or when $q=\infty$ ?

We obtain a partial positive result later. Namely, we show later that if $\Omega \subset \mathbf{R}^{n}$ is a bounded open set, then $\operatorname{cap}_{(p, 1)}(\cdot, \Omega)$ is a Choquet capacity whenever $1 \leq n<$ $p<\infty$ or $1<n=p<\infty$.

Remark 5.4. Suppose $1 \leq q \leq \infty$. The definition of the $(p, q)$-capacity easily implies

whenever $K$ is a compact set in $\Omega$.

$$
\operatorname{cap}_{(p, q)}(K, \Omega)=\operatorname{cap}_{(p, q)}(\partial K, \Omega)
$$

5.2. The Sobolev-Lorentz $\boldsymbol{p}, \boldsymbol{q}$ relative capacity. Let $n \geq 1$ be an integer. Suppose $1<p<\infty$ and $1 \leq q \leq \infty$. We can introduce the $p, q$ relative capacity the way we introduced the $(p, q)$ relative capacity. Let $\Omega \subset \mathbf{R}^{n}$ be a bounded and open set and let $E$ be a subset of $\Omega$. We can define the Sobolev-Lorentz $p, q$ relative capacity of the pair $(E, \Omega)$ by

$$
\operatorname{cap}_{p, q}(E, \Omega)=\inf \left\{\|\nabla u\|_{L^{p, q}\left(\Omega ; \mathbf{R}^{n}\right)}^{p}: u \in \mathcal{A}(E, \Omega)\right\}
$$

where (like before)

$$
\mathcal{A}(E, \Omega)=\left\{u \in H_{0}^{1,(p, q)}(\Omega): u \geq 1 \text { in a neighborhood of } E\right\} .
$$

Like before, we call $\mathcal{A}(E, \Omega)$ the set of admissible functions for the condenser $(E, \Omega)$. If $\mathcal{A}(E, \Omega)=\emptyset$, we set $\operatorname{cap}_{p, q}(E, \Omega)=\infty$.

Since $H_{0}^{1,(p, q)}(\Omega)$ is closed under truncations from below by 0 and from above by 1 and since these truncations do not increase the $p, q$-quasinorm of the distributional gradients, it is enough to consider only those admissible functions $u$ for which $0 \leq$ $u \leq 1$.

5.2.1. Basic properties of the $\boldsymbol{p}, \boldsymbol{q}$ relative capacity. Usually, a capacity is a monotone and subadditive set function. The following theorem will show, among other things, that this is true in the case of the $p, q$ relative capacity. In our book [6] we studied the case $1<p<\infty$ and $n \geq 2$. We extend these results to the case $n=1$. The following theorem generalizes Theorem 3.2 from Costea-Maz'ya [8] and Theorem 4.2.2 from [6].

Theorem 5.5. (See Costea-Maz'ya [8, Theorem 3.2] and [6, Theorem 4.2.2]) Let $n \geq 1$ be an integer. Suppose $1<p<\infty$ and $1 \leq q \leq \infty$. Let $\Omega \subset \mathbf{R}^{n}$ be a bounded open set. The set function $E \mapsto \operatorname{cap}_{p, q}(E, \Omega), E \subset \Omega$, enjoys the following properties:

(i) If $E_{1} \subset E_{2} \subset \Omega$, then $\operatorname{cap}_{p, q}\left(E_{1}, \Omega\right) \leq \operatorname{cap}_{p, q}\left(E_{2}, \Omega\right)$.

(ii) If $\Omega_{1} \subset \Omega_{2} \subset \mathbf{R}^{n}$ are open and $E \subset \Omega_{1}$, then

$$
\operatorname{cap}_{p, q}\left(E, \Omega_{2}\right) \leq \operatorname{cap}_{p, q}\left(E, \Omega_{1}\right) .
$$

(iii) $\operatorname{cap}_{p, q}(E, \Omega)=\inf \left\{\operatorname{cap}_{p, q}(U, \Omega): E \subset U \subset \Omega, U\right.$ open $\}$. 
(iv) If $K_{i}$ is a decreasing sequence of compact subsets of $\Omega$ with $K=\bigcap_{i=1}^{\infty} K_{i}$, then

$$
\operatorname{cap}_{p, q}(K, \Omega)=\lim _{i \rightarrow \infty} \operatorname{cap}_{p, q}\left(K_{i}, \Omega\right) .
$$

(v) Suppose that $1<q \leq p$. If $E_{1} \subset E_{2} \subset \ldots \subset E=\bigcup_{i=1}^{\infty} E_{i} \subset \Omega$, then

$$
\operatorname{cap}_{p, q}(E, \Omega)=\lim _{i \rightarrow \infty} \operatorname{cap}_{p, q}\left(E_{i}, \Omega\right) .
$$

(vi) Suppose that $1 \leq q \leq p$. If $E=\bigcup_{i=1}^{\infty} E_{i} \subset \Omega$, then

$$
\operatorname{cap}_{p, q}(E, \Omega)^{q / p} \leq \sum_{i=1}^{\infty} \operatorname{cap}_{p, q}\left(E_{i}, \Omega\right)^{q / p} \text {. }
$$

(vii) Suppose that $p<q<\infty$. If $E=\bigcup_{i=1}^{\infty} E_{i} \subset \Omega$, then

$$
\operatorname{cap}_{p, q}(E, \Omega) \leq \sum_{i=1}^{\infty} \operatorname{cap}_{p, q}\left(E_{i}, \Omega\right) \text {. }
$$

(viii) Suppose that $q=\infty$. Let $k \geq 1$ be an integer. If $E=\bigcup_{i=1}^{k} E_{i} \subset \Omega$, then

$$
\operatorname{cap}_{p, q}(E, \Omega) \leq \sum_{i=1}^{k} \operatorname{cap}_{p, q}\left(E_{i}, \Omega\right) .
$$

(ix) Suppose that $1 \leq q \leq \infty$. If $\Omega_{1}$ and $\Omega_{2}$ are two disjoint open sets and $E \subset \Omega_{1}$, then

$$
\operatorname{cap}_{p, q}\left(E, \Omega_{1} \cup \Omega_{2}\right)=\operatorname{cap}_{p, q}\left(E, \Omega_{1}\right) .
$$

(x) Suppose that $1 \leq q \leq p$. Suppose that $\Omega_{1}, \ldots, \Omega_{k}$ are pairwise disjoint open sets and $E_{i}$ are subsets of $\Omega_{i}$ for $i=1, \ldots, k$. If $E=\bigcup_{i=1}^{k} E_{i}$ and $\Omega=\bigcup_{i=1}^{k} \Omega_{i}$, then

$$
\operatorname{cap}_{p, q}(E, \Omega) \geq \sum_{i=1}^{k} \operatorname{cap}_{p, q}\left(E_{i}, \Omega_{i}\right) .
$$

(xi) Suppose that $p<q<\infty$. Suppose that $\Omega_{i}, \ldots, \Omega_{k}$ are pairwise disjoint open sets and $E_{i}$ are subsets of $\Omega_{i}$ for $i=1, \ldots, k$. If $E=\bigcup_{i=1}^{k} E_{i}$ and $\Omega=\bigcup_{i=1}^{k} \Omega_{i}$, then

$$
\operatorname{cap}_{p, q}(E, \Omega)^{q / p} \geq \sum_{i=1}^{k} \operatorname{cap}_{p, q}\left(E_{i}, \Omega_{i}\right)^{q / p} .
$$

Proof. This result was proved in [6] for $n \geq 2$. See [6, Theorem 4.2.2]. The proof of the case $n=1$ is very similar to the proof of [6, Theorem 4.2.2] and omitted.

The set function $\operatorname{cap}_{p, q}(\cdot, \Omega)$ satisfies properties (i), (iv), and (v) of Theorem 5.5 whenever $1<q \leq p<\infty$ and $\Omega$ is a bounded open set in $\mathbf{R}^{n}$, where $n \geq 1$ is an integer. Thus, $\operatorname{cap}_{(p, q)}(\cdot, \Omega)$ is a Choquet capacity (relative to $\Omega$ ) whenever $1<q \leq p<\infty$ and $\Omega$ is a bounded open set in $\mathbf{R}^{n}$, where $n \geq 1$ is an integer. We may thus invoke an important capacitability theorem of Choquet and state the following result. See Doob [9, Appendix II].

Theorem 5.6. Let $\Omega$ be a bounded open set in $\mathbf{R}^{n}$, where $n \geq 1$ is an integer. Suppose $1<q \leq p<\infty$. The set function $E \mapsto \operatorname{cap}_{p, q}(E, \Omega), E \subset \Omega$, is a Choquet capacity. In particular, all Borel subsets (in fact, all analytic) subsets $E$ of $\Omega$ are capacitable, i.e.

$$
\operatorname{cap}_{p, q}(E, \Omega)=\sup \left\{\operatorname{cap}_{p, q}(K, \Omega): K \subset E \text { compact }\right\} .
$$


The set function $\operatorname{cap}_{p, q}(\cdot, \Omega)$ satisfies properties (i) and (iv) of Theorem 5.5 whenever $q=1$ or $p<q \leq \infty$. Like in Theorem 5.5, the set $\Omega$ is bounded and open in $\mathbf{R}^{n}$, where $n \geq 1$ is an integer.

Question 5.7. Let $1<p<\infty$ be fixed. Suppose that $\Omega$ is a bounded open set in $\mathbf{R}^{n}$, where $n \geq 1$ is an integer. Is $\operatorname{cap}_{p, q}(\cdot, \Omega)$ a Choquet capacity when $q=1$ or when $p<q \leq \infty$ ?

We obtain a partial positive result later. Namely, we show later that if $\Omega \subset \mathbf{R}^{n}$ is a bounded open set, then $\operatorname{cap}_{p, 1}(\cdot, \Omega)$ is a Choquet capacity whenever $1 \leq n<p<\infty$ or $1<n=p<\infty$.

Remark 5.8. The definition of the $p, q$-capacity easily implies

$$
\operatorname{cap}_{p, q}(K, \Omega)=\operatorname{cap}_{p, q}(\partial K, \Omega)
$$

whenever $K$ is a compact set in $\Omega$.

5.3. The Sobolev-Lorentz $(\boldsymbol{p}, \boldsymbol{q})$ global capacity. Let $n \geq 1$ be an integer. Suppose $1<p<\infty$ and $1 \leq q \leq \infty$. For a set $E \subset \mathbf{R}^{n}$ we define the Sobolev-Lorentz global $(p, q)$-capacity of $E$ by

$$
\operatorname{Cap}_{(p, q)}(E)=\inf \|u\|_{H^{1,(p, q)}\left(\mathbf{R}^{n}\right)}^{p},
$$

where $u$ runs through the set

$$
S(E)=\left\{u \in H_{0}^{1,(p, q)}\left(\mathbf{R}^{n}\right): u \geq 1 \text { in an open set containing } E\right\} .
$$

If $S(E)=\emptyset$, we set $\operatorname{Cap}_{(p, q)}(E)=\infty$. It is obvious that the same number is obtained if the infimum in the definition is taken over $u \in S(E)$ with $0 \leq u \leq 1$.

5.3.1. Basic properties of the $(p, q)$ global capacity. The following theorem summarizes the properties of the global Sobolev-Lorentz $(p, q)$-capacity, extending our results from [6, Theorem 4.6.2] to the case $n=1$.

Theorem 5.9. (See [6, Theorem 4.6.2]) Let $n \geq 1$ be an integer. Suppose $1<p<\infty$ and $1 \leq q \leq \infty$. The set function $E \mapsto \operatorname{Cap}_{(p, q)}(E), E \subset \mathbf{R}^{n}$, has the following properties:

(i) If $E_{1} \subset E_{2}$, then $\operatorname{Cap}_{(p, q)}\left(E_{1}\right) \leq \operatorname{Cap}_{(p, q)}\left(E_{2}\right)$.

(ii) $\operatorname{Cap}_{(p, q)}(E)=\inf \left\{\operatorname{Cap}_{(p, q)}(U): E \subset U \subset \mathbf{R}^{n}, U\right.$ open $\}$.

(iii) If $K_{i}$ is a decreasing sequence of compact subsets of $\mathbf{R}^{n}$ with $K=\bigcap_{i=1}^{\infty} K_{i}$, then

$$
\operatorname{Cap}_{(p, q)}(K)=\lim _{i \rightarrow \infty} \operatorname{Cap}_{(p, q)}\left(K_{i}\right) .
$$

(iv) Suppose that $1<q<\infty$. If $E_{1} \subset E_{2} \subset \ldots \subset E=\bigcup_{i=1}^{\infty} E_{i} \subset \mathbf{R}^{n}$, then

$$
\operatorname{Cap}_{(p, q)}(E)=\lim _{i \rightarrow \infty} \operatorname{Cap}_{(p, q)}\left(E_{i}\right) \text {. }
$$

(v) If $E=\bigcup_{i=1}^{\infty} E_{i}$, then

$$
\operatorname{Cap}_{(p, q)}(E)^{1 / p} \leq \sum_{i=1}^{\infty} \operatorname{Cap}_{(p, q)}\left(E_{i}\right)^{1 / p}
$$

Proof. This result was proved in [6] for $n \geq 2$. See [6, Theorem 4.6.2]. The proof of the case $n=1$ is very similar to the proof of [6, Theorem 4.6.2] and omitted.

The set function $\operatorname{Cap}_{(p, q)}(\cdot)$ satisfies properties (i), (iii), and (iv) of Theorem 5.9 whenever $n \geq 1$ is an integer and $1<p, q<\infty$. Thus, this set function is a Choquet capacity whenever $n \geq 1$ is an integer and $1<p, q<\infty$. We may thus invoke 
an important capacitability theorem of Choquet and state the following result. See Doob [9, Appendix II].

Theorem 5.10. Let $n \geq 1$ be an integer. Suppose $1<p, q<\infty$. The set function $E \mapsto \operatorname{Cap}_{(p, q)}(E), E \subset \mathbf{R}^{n}$, is a Choquet capacity. In particular, all Borel subsets (in fact, all analytic) subsets $E$ of $\mathbf{R}^{n}$ are capacitable, i.e.

$$
\operatorname{Cap}_{(p, q)}(E)=\sup \left\{\operatorname{Cap}_{(p, q)}(K): K \subset E \text { compact }\right\} .
$$

The set function $\operatorname{Cap}_{(p, q)}(\cdot)$ satisfies properties (i) and (iii) of Theorem 5.9 whenever $q=1$ or $q=\infty$. Like in Theorem $5.9, n \geq 1$ is an integer.

Question 5.11. Let $n \geq 1$ be an integer. Suppose $1<p<\infty$. Is $\operatorname{Cap}_{(p, q)}(\cdot)$ Choquet when $q=1$ or when $q=\infty$ ?

We obtain a partial positive result later. Namely, we show later that $\operatorname{Cap}_{(p, 1)}(\cdot)$ is a Choquet capacity whenever $1 \leq n<p<\infty$ or $1<n=p<\infty$.

5.4. The Sobolev-Lorentz $\boldsymbol{p}, \boldsymbol{q}$ global capacity. Let $n \geq 1$ be an integer. Suppose $1<p<\infty$ and $1 \leq q \leq \infty$. We can introduce the global $p, q$-capacity the way we introduced the global $(p, q)$-capacity. For a set $E \subset \mathbf{R}^{n}$ we define the Sobolev-Lorentz global $p, q$-capacity of $E$ by

$$
\operatorname{Cap}_{p, q}(E)=\inf \|u\|_{H^{1, p, q}\left(\mathbf{R}^{n}\right)}^{p},
$$

where $u$ runs through the set

$$
S(E)=\left\{u \in H_{0}^{1,(p, q)}\left(\mathbf{R}^{n}\right): u \geq 1 \text { in an open set containing } E\right\} .
$$

If $S(E)=\emptyset$, we set $\operatorname{Cap}_{p, q}(E)=\infty$. It is obvious that the same number is obtained if the infimum in the definition is taken over $u \in S(E)$ with $0 \leq u \leq 1$.

5.4.1. Basic properties of the $\boldsymbol{p}, \boldsymbol{q}$ global capacity. The following theorem summarizes the properties of the global Sobolev-Lorentz $p, q$-capacity, extending our results from [6, Theorem 4.7.3] to the case $n=1$.

Theorem 5.12. (See [6, Theorem 4.7.3]) Let $n \geq 1$ be an integer. Suppose $1<p<\infty$ and $1 \leq q \leq \infty$. The set function $E \mapsto \operatorname{Cap}_{p, q}(E), E \subset \mathbf{R}^{n}$, enjoys the following properties:

(i) If $E_{1} \subset E_{2}$, then $\operatorname{Cap}_{p, q}\left(E_{1}\right) \leq \operatorname{Cap}_{p, q}\left(E_{2}\right)$.

(ii) $\operatorname{Cap}_{p, q}(E)=\inf \left\{\operatorname{Cap}_{p, q}(U): E \subset U \subset \mathbf{R}^{n}, U\right.$ open $\}$.

(iii) If $K_{i}$ is a decreasing sequence of compact subsets of $\mathbf{R}^{n}$ with $K=\bigcap_{i=1}^{\infty} K_{i}$, then

$$
\operatorname{Cap}_{p, q}(K)=\lim _{i \rightarrow \infty} \operatorname{Cap}_{p, q}\left(K_{i}\right) .
$$

(iv) Suppose that $1<q \leq p$. If $E_{1} \subset E_{2} \subset \ldots \subset E=\bigcup_{i=1}^{\infty} E_{i} \subset \mathbf{R}^{n}$, then

$$
\operatorname{Cap}_{p, q}(E)=\lim _{i \rightarrow \infty} \operatorname{Cap}_{p, q}\left(E_{i}\right) \text {. }
$$

(v) Suppose that $1 \leq q \leq p$. If $E=\bigcup_{i=1}^{\infty} E_{i} \subset \mathbf{R}^{n}$, then

$$
\operatorname{Cap}_{p, q}(E)^{q / p} \leq \sum_{i=1}^{\infty} \operatorname{Cap}_{p, q}\left(E_{i}\right)^{q / p} \text {. }
$$

(vi) Suppose that $p<q<\infty$. If $E=\bigcup_{i=1}^{\infty} E_{i} \subset \mathbf{R}^{n}$, then

$$
\operatorname{Cap}_{p, q}(E) \leq \sum_{i=1}^{\infty} \operatorname{Cap}_{p, q}\left(E_{i}\right) \text {. }
$$


(vii) Suppose that $q=\infty$. Let $k \geq 1$ be an integer. If $E=\bigcup_{i=1}^{k} E_{i} \subset \mathbf{R}^{n}$, then

$$
\operatorname{Cap}_{p, q}(E) \leq \sum_{i=1}^{k} \operatorname{Cap}_{p, q}\left(E_{i}\right) \text {. }
$$

Proof. This result was proved in [6] for $n \geq 2$. See [6, Theorem 4.7.3]. The proof of the case $n=1$ is very similar to the proof of [6, Theorem 4.7.3] and omitted.

The set function $\operatorname{Cap}_{p, q}(\cdot)$ satisfies properties (i), (iii), and (iv) of Theorem 5.12 whenever $n \geq 1$ is an integer and $1<q \leq p<\infty$. Thus, this set function is a Choquet capacity whenever $n \geq 1$ is an integer and $1<q \leq p<\infty$. We may thus invoke an important capacitability theorem of Choquet and state the following result. See Doob [9, Appendix II].

Theorem 5.13. Let $n \geq 1$ be an integer. Suppose $1<q \leq p<\infty$. The set function $E \mapsto \operatorname{Cap}_{p, q}(E), E \subset \mathbf{R}^{n}$, is a Choquet capacity. In particular, all Borel subsets (in fact, all analytic) subsets $E$ of $\mathbf{R}^{n}$ are capacitable, i.e.

$$
\operatorname{Cap}_{p, q}(E)=\sup \left\{\operatorname{Cap}_{p, q}(K): K \subset E \text { compact }\right\} \text {. }
$$

The set function $\operatorname{Cap}_{p, q}(\cdot)$ satisfies properties (i) and (iii) of Theorem 5.12 whenever $q=1$ or $p<q \leq \infty$. Like in Theorem 5.12, $n \geq 1$ is an integer.

Question 5.14. Let $n \geq 1$ be an integer. Suppose $1<p<\infty$. Is $\operatorname{Cap}_{p, q}(\cdot)$ a Choquet capacity when $q=1$ or when $p<q \leq \infty$ ?

We obtain a partial positive result later. Namely, we show later that $\operatorname{Cap}_{p, 1}(\cdot)$ is a Choquet capacity whenever $1 \leq n<p<\infty$ or $1<n=p<\infty$.

\section{Sharp estimates for the Sobolev-Lorentz $n, 1$ relative capacity}

In [5] we studied the $n, q$ relative capacity for $n \geq 2$ and $1 \leq q \leq \infty$ and we obtained sharp estimates for the $n, q$ relative capacity of the condensers $(\bar{B}(0, r), B(0,1))$ for small values of $r$ in $[0,1)$. See [5, Theorem 3.11]. In this section we obtain a few new results. For instance, we obtain sharp estimates for the $n, 1$ relative capacity of the aforementioned concentric condensers for ALL $r$ in $[0,1)$. See Theorem 6.2 (i). Thus, we improve the estimates that we obtained in [5] for the $n, 1$ relative capacity. In particular, we obtain the exact value for the $n, 1$ capacity of a point relative to all its bounded open neighborhoods from $\mathbf{R}^{n}$, a strictly positive number as we saw in [5, Corollary 3.8]. See Theorem 6.2 (ii).

Moreover, we obtain a new result concerning the $n, 1$ global capacity. We show that this aforementioned value is also the value of the global $n, 1$ capacity of any point from $\mathbf{R}^{n}$. See Theorem 6.3. This constant will also come into play later when we give a new proof of the embedding $H_{0}^{1,(n, 1)}(\Omega) \hookrightarrow C(\bar{\Omega}) \cap L^{\infty}(\Omega)$, where $\Omega \subset \mathbf{R}^{n}$ is open and $n \geq 2$ is an integer. See Theorem 6.4. This embedding is proved by using the exact value of the $n, 1$ capacity of a point relative to any of its bounded open neighborhoods from $\mathbf{R}^{n}$.

In order to obtain the sharp estimates for the condensers $(\bar{B}(0, r), B(0,1))$ for all $r$ in [0,1), we revisit Proposition 2.11 from [5] for $p=n>1$ and $q=1$.

Proposition 6.1. Suppose $n \geq 2$ is an integer. Let $0 \leq r<1$ be fixed. Let $w:\left[\Omega_{n} r^{n}, \Omega_{n}\right] \rightarrow[0, \infty)$ be defined by $w(t)=\left(t / \Omega_{n}\right)^{1 / n}$. Suppose $f:[r, 1] \rightarrow[0, \infty)$ is continuous and let $g:\left[\Omega_{n} r^{n}, \Omega_{n}\right] \rightarrow[0, \infty)$ be defined by $g(t)=f(w(t))$. Then

$$
\|g\|_{L^{n, 1}\left(\left[\Omega_{n} r^{n}, \Omega_{n}\right]\right)} \geq n \Omega_{n}^{1 / n}\left(1-r^{n}\right)^{-1 / n^{\prime}}\|f\|_{L^{1}([r, 1])} .
$$


Proof. By applying [5, Proposition 2.11] for $p=n>1$ and $q=1$, we obtain

$$
\begin{aligned}
\|g\|_{L^{n, 1}\left(\left[\Omega_{n} r^{n}, \Omega_{n}\right]\right)} & \geq n \Omega_{n}\left\|\left(t / \Omega_{n}\right)^{-1 / n^{\prime}}\right\|_{L^{n^{\prime}, \infty}\left(\left[\Omega_{n} r^{n}, \Omega_{n}\right]\right)}^{-1}\|f\|_{L^{1}([r, 1])} \\
& =n \Omega_{n}^{1 / n}\left\|t^{-1 / n^{\prime}}\right\|_{L^{n^{\prime}, \infty}\left(\left[\Omega_{n} r^{n}, \Omega_{n}\right]\right)}^{-1}\|f\|_{L^{1}([r, 1])} .
\end{aligned}
$$

We compute $\left\|t^{-1 / n^{\prime}}\right\|_{L^{n^{\prime}, \infty}\left(\left[\Omega_{n} r^{n}, \Omega_{n}\right]\right)}$. We notice that

$$
\left\|t^{-1 / n^{\prime}}\right\|_{L^{n^{\prime}, \infty}\left(\left[\Omega_{n} r^{n}, \Omega_{n}\right]\right)}=\left\|\left(t+\Omega_{n} r^{n}\right)^{-1 / n^{\prime}}\right\|_{L^{n^{\prime}, \infty}\left(\left[0, \Omega_{n}\left(1-r^{n}\right)\right]\right)} .
$$

An easy computation shows that

$$
\begin{aligned}
\left\|\left(t+\Omega_{n} r^{n}\right)^{-1 / n^{\prime}}\right\|_{L^{n^{\prime}, \infty}\left(\left[0, \Omega_{n}\left(1-r^{n}\right)\right]\right)} & =\sup _{0 \leq t \leq \Omega_{n}\left(1-r^{n}\right)} t^{1 / n^{\prime}}\left(t+\Omega_{n} r^{n}\right)^{-1 / n^{\prime}} \\
& =\sup _{0 \leq t \leq \Omega_{n}\left(1-r^{n}\right)}\left(\frac{t}{t+\Omega_{n} r^{n}}\right)^{1 / n^{\prime}}=\left(1-r^{n}\right)^{1 / n^{\prime}} .
\end{aligned}
$$

This finishes the proof.

Next we obtain sharp estimates for the $n, 1$ relative capacity of the condensers $(\bar{B}(0, r), B(0,1))$ for ALL $r$ in $[0,1)$. Thus, we improve the estimates obtained in [5, Theorem 3.11] for the $n, 1$ relative capacity of the aforementioned concentric condensers. As a consequence we obtain the exact value of the $n, 1$ capacity of a point relative to all its bounded open neighborhoods from $\mathbf{R}^{n}$.

Theorem 6.2. Let $n \geq 2$ be an integer.

(i) We have

$$
n^{n} \Omega_{n}\left(1-r^{n}\right)^{1-n} \leq \operatorname{cap}_{n, 1}(\bar{B}(0, r), B(0,1)) \leq n^{n} \Omega_{n} \frac{1-r^{n}}{(1-r)^{n}}
$$

for every $0 \leq r<1$.

(ii) We have

$$
\operatorname{cap}_{n, 1}(\{x\}, \Omega)=n^{n} \Omega_{n}
$$

whenever $x \in \mathbf{R}^{n}$ and $\Omega$ is a bounded open set in $\mathbf{R}^{n}$ containing $x$.

Proof. We start by proving claim (i). Let $r \in[0,1)$ be fixed. We want to compute the lower estimate. In order to do that, it is enough to consider via [5, Lemma 3.6] only the admissible radial functions in $C_{0}^{\infty}(B(0,1))$ that are 1 on a neighborhood of $\bar{B}(0, r)$. Let $u$ be such a function. There exists a function $f \in C^{\infty}([0,1])$ such that $u(x)=f(|x|)$ for every $x \in B(0,1)$. Hence $|\nabla u(x)|=\left|f^{\prime}\right|(|x|)$ for every $x \in$ $B(0,1)$. Moreover, $f^{\prime}(t)=0$ for every $t \in[0, r]$. If we define $g:\left[0, \Omega_{n}\right] \rightarrow[0, \infty)$ by $g(t)=\left|f^{\prime}\right|\left(\left(t / \Omega_{n}\right)^{1 / n}\right)$, we notice that $g$ is a continuous function compactly supported in $\left(\Omega_{n} r^{n}, \Omega_{n}\right)$. Moreover, since $|\nabla u(x)|=g\left(\Omega_{n}|x|^{n}\right)$ for every $x \in B(0,1)$, it follows that $|\nabla u|$ and $g$ have the same distribution function. From this and the fact that $g$ is supported in $\left(\Omega_{n} r^{n}, \Omega_{n}\right)$, we obtain

$$
\|\nabla u\|_{L^{n, 1}\left(B(0,1) ; \mathbf{R}^{n}\right)}=\|g\|_{L^{n, 1}\left(\left[\Omega_{n} r^{n}, \Omega_{n}\right]\right)} .
$$

But via Proposition 6.1 we have

$$
\|g\|_{L^{n, 1}\left(\left[\Omega_{n} r^{n}, \Omega_{n}\right]\right)} \geq n \Omega_{n}^{1 / n}\left(1-r^{n}\right)^{-1 / n^{\prime}}\left\|f^{\prime}\right\|_{L^{1}([r, 1])}
$$

and since $\left\|f^{\prime}\right\|_{L^{1}([r, 1])} \geq f(r)-f(1)=1$, we obtain

$$
\|\nabla u\|_{L^{n, 1}\left(B(0,1) ; \mathbf{R}^{n}\right)}=\|g\|_{L^{n, 1}\left(\left[\Omega_{n} r^{n}, \Omega_{n}\right]\right)} \geq n \Omega_{n}^{1 / n}\left(1-r^{n}\right)^{-1 / n^{\prime}} .
$$

By taking the infimum over all admissible radial functions that are in $C_{0}^{\infty}(B(0,1))$, we obtain the desired lower estimate. 
Now we compute the upper estimate. Let $u_{r}: B(0,1) \rightarrow[0,1]$ be defined by

$$
u_{r}(x)= \begin{cases}1, & \text { if } 0 \leq|x| \leq r, \\ \frac{1-|x|}{1-r}, & \text { if } r<|x|<1 .\end{cases}
$$

We notice that $u_{r}$ is a Lipschitz function on $B(0,1)$ that can be extended continuously by 0 on $\partial B(0,1)$. Thus, $u_{r}$ in $H_{0}^{1,(n, 1)}(B(0,1))$. Moreover, the function $\frac{1}{1-\varepsilon} u_{r}$ is admissible for the condenser $(\bar{B}(0, r), B(0,1))$ for every $\varepsilon \in(0,1)$.

Thus, we have

$$
\operatorname{cap}_{n, 1}(\bar{B}(0, r), B(0,1)) \leq \frac{1}{(1-\varepsilon)^{n}}\left\|\nabla u_{r}\right\|_{L^{n, 1}\left(B(0,1) ; \mathbf{R}^{n}\right)}^{n}
$$

for every $\varepsilon \in(0,1)$. By letting $\varepsilon \rightarrow 0$, the above inequality yields

$$
\operatorname{cap}_{n, 1}(\bar{B}(0, r), B(0,1)) \leq\left\|\nabla u_{r}\right\|_{L^{n, 1}\left(B(0,1) ; \mathbf{R}^{n}\right)}^{n} .
$$

An easy computation shows that

$$
\left|\nabla u_{r}(x)\right|= \begin{cases}0 & \text { if } 0 \leq|x|<r \\ \frac{1}{1-r} & \text { if } r<|x|<1\end{cases}
$$

Thus,

$$
\begin{aligned}
\left\|\nabla u_{r}\right\|_{L^{n, 1}\left(B(0,1) ; \mathbf{R}^{n}\right)} & =\frac{1}{1-r}\left\|\chi_{B(0,1) \backslash \bar{B}(0, r)}\right\|_{L^{n, 1}(B(0,1))}=\frac{1}{1-r} \int_{0}^{\Omega_{n}\left(1-r^{n}\right)} t^{\frac{1}{n}-1} d t \\
& =\frac{1}{1-r} n\left[\Omega_{n}\left(1-r^{n}\right)\right]^{1 / n}=n \Omega_{n}^{1 / n} \frac{\left(1-r^{n}\right)^{1 / n}}{1-r} .
\end{aligned}
$$

Hence, we obtain the desired upper estimate

$$
\operatorname{cap}_{n, 1}(\bar{B}(0, r), B(0,1)) \leq n^{n} \Omega_{n} \frac{\left(1-r^{n}\right)}{(1-r)^{n}} .
$$

We obtained the desired lower and upper estimates for $\operatorname{cap}_{n, 1}(\bar{B}(0, r), B(0,1))$. This finishes the proof of claim (i).

We prove claim (ii) now. For $r=0$, claim (i) yields

$$
\operatorname{cap}_{n, 1}(\{0\}, B(0,1))=n^{n} \Omega_{n} .
$$

From this, the invariance of the $n, 1$ relative capacity under translations, [5, Lemma 3.4] and [5, Theorem 3.2 (ii)] (see also Theorem 5.5 (ii)), the desired conclusion follows. This finishes the proof.

6.1. Sharp estimates for the $\boldsymbol{n}, 1$ global capacity. In Theorem 6.2 we computed the exact value of the $n, 1$ capacity of a point relative to all its bounded open neighborhoods from $\mathbf{R}^{n}$. We now prove that this strictly positive number is also the exact value of the $n, 1$ global capacity of any point from $\mathbf{R}^{n}$.

Theorem 6.3. Let $n \geq 2$ be an integer. We have

$$
\operatorname{Cap}_{n, 1}(\{x\})=n^{n} \Omega_{n}>0
$$

for every $x \in \mathbf{R}^{n}$.

Proof. Since the $n, 1$ global capacity is invariant under translation, it is enough to prove via Theorem 6.2 (ii) that

$$
\operatorname{Cap}_{n, 1}(\{0\})=n^{n} \Omega_{n} .
$$


We prove first that

$$
n^{n} \Omega_{n} \leq \operatorname{Cap}_{n, 1}(\{0\}) .
$$

Fix $\varepsilon>0$. Let $u \in S(\{0\})$ be an admissible function for $\{0\}$ with respect to the $n, 1$ global capacity such that

$$
\|u\|_{L^{n, 1}\left(\mathbf{R}^{n}\right)}+\|\nabla u\|_{L^{n, 1}\left(\mathbf{R}^{n} ; \mathbf{R}^{n}\right)}<\operatorname{Cap}_{n, 1}(\{0\})^{1 / n}+\varepsilon .
$$

Without loss of generality we can assume that $u \in S(\{0\}) \cap C_{0}^{\infty}\left(\mathbf{R}^{n}\right)$. Then $u$ is compactly supported in a bounded open set $U \subset \mathbf{R}^{n}$ that contains the origin. It is easy to see that $S(\{0\}) \cap C_{0}^{\infty}(U)=\mathcal{A}(\{0\}, U) \cap C_{0}^{\infty}(U)$. Thus, $u \in \mathcal{A}(\{0\}, U)$.

Therefore we have via Theorem 6.2 (ii)

$$
\begin{aligned}
n \Omega_{n}^{1 / n} & =\operatorname{cap}_{n, 1}(\{0\}, U)^{1 / n} \leq\|\nabla u\|_{L^{n, 1}\left(U ; \mathbf{R}^{n}\right)}=\|\nabla u\|_{L^{n, 1}\left(\mathbf{R}^{n} ; \mathbf{R}^{n}\right)} \\
& \leq\|u\|_{L^{n, 1}\left(\mathbf{R}^{n}\right)}+\|\nabla u\|_{L^{n, 1}\left(\mathbf{R}^{n} ; \mathbf{R}^{n}\right)}<\operatorname{Cap}_{n, 1}(\{0\})^{1 / n}+\varepsilon .
\end{aligned}
$$

By letting $\varepsilon \rightarrow 0$, we see that indeed $n^{n} \Omega_{n} \leq \operatorname{Cap}_{n, 1}(\{0\})$.

Conversely, we want to show that $\operatorname{Cap}_{n, 1}(\{0\}) \leq n^{n} \Omega_{n}$. Let $u: \mathbf{R}^{n} \rightarrow[0,1]$ be defined by

$$
u(x)= \begin{cases}1-|x| & \text { if } 0 \leq|x| \leq 1 \\ 0 & \text { if }|x|>1\end{cases}
$$

We notice that $u$ is a Lipschitz function on $\mathbf{R}^{n}$ that is supported in $\bar{B}(0,1)$. Thus, $u \in H_{0}^{1,(n, 1)}\left(\mathbf{R}^{n}\right)$. Moreover, the function $\frac{1}{1-\varepsilon} u$ is in $S(\{0\})$ for every $\varepsilon$ in $(0,1)$. For every $r$ in $(0, \infty)$ we define $u_{r}: \mathbf{R}^{n} \rightarrow[0,1]$ by $u_{r}(x)=u\left(\frac{x}{r}\right)$. We notice that $u_{r}$ is a Lipschitz function on $\mathbf{R}^{n}$ that is supported in $\bar{B}(0, r)$ for every $r>0$. Thus, $u_{r} \in H_{0}^{1,(n, 1)}\left(\mathbf{R}^{n}\right)$ for every $r>0$. Moreover, the function $\frac{1}{1-\varepsilon} u_{r}$ is in $S(\{0\})$ for every $r>0$ and for every $\varepsilon$ in $(0,1)$.

It is easy to see that

$$
\left\|u_{r}\right\|_{L^{n, 1}\left(\mathbf{R}^{n}\right)}=r\|u\|_{L^{n, 1}\left(\mathbf{R}^{n}\right)} \text { and }\left\|\nabla u_{r}\right\|_{L^{n, 1}\left(\mathbf{R}^{n} ; \mathbf{R}^{n}\right)}=\|\nabla u\|_{L^{n, 1}\left(\mathbf{R}^{n} ; \mathbf{R}^{n}\right)}
$$

for every $r>0$. Thus,

$$
\begin{aligned}
\operatorname{Cap}_{n, 1}(\{0\})^{1 / n} & \leq \frac{1}{1-\varepsilon}\left(\left\|u_{r}\right\|_{L^{n, 1}\left(\mathbf{R}^{n}\right)}+\left\|\nabla u_{r}\right\|_{L^{n, 1}\left(\mathbf{R}^{n} ; \mathbf{R}^{n}\right)}\right) \\
& =\frac{1}{1-\varepsilon}\left(r\|u\|_{L^{n, 1}\left(\mathbf{R}^{n}\right)}+\|\nabla u\|_{L^{n, 1}\left(\mathbf{R}^{n} ; \mathbf{R}^{n}\right)}\right)
\end{aligned}
$$

for every $r>0$ and for every $\varepsilon$ in $(0,1)$. By letting $r \rightarrow 0$ and $\varepsilon \rightarrow 0$, we obtain

$$
\operatorname{Cap}_{n, 1}(\{0\}) \leq\|\nabla u\|_{L^{n, 1}\left(\mathbf{R}^{n} ; \mathbf{R}^{n}\right)}^{n} .
$$

An easy computation shows that

$$
|\nabla u(x)|= \begin{cases}1 & \text { if } 0<|x|<1 \\ 0 & \text { if }|x|>1\end{cases}
$$

Thus,

$$
\|\nabla u\|_{L^{n, 1}\left(\mathbf{R}^{n} ; \mathbf{R}^{n}\right)}=\left\|\chi_{B(0,1)}\right\|_{L^{n, 1}\left(\mathbf{R}^{n}\right)}=\int_{0}^{\Omega_{n}} t^{\frac{1}{n}-1} d t=n \Omega_{n}^{1 / n} .
$$

Therefore, we obtain

$$
\operatorname{Cap}_{n, 1}(\{0\}) \leq\|\nabla u\|_{L^{n, 1}\left(\mathbf{R}^{n} ; \mathbf{R}^{n}\right)}^{n}=n^{n} \Omega_{n} .
$$

This finishes the proof of the theorem. 
The following theorem gives a new proof of the embedding $H_{0}^{1,(n, 1)}(\Omega) \hookrightarrow C(\bar{\Omega}) \cap$ $L^{\infty}(\Omega)$ whenever $\Omega \subset \mathbf{R}^{n}$ is an open set and $n \geq 2$. This embedding was proved before by Stein in [17] and by Cianchi-Pick in [2]. See [2, Theorem 3.5 (i)]. Our approach is different. Our proof uses the theory of the $n, 1$ relative capacity in $\mathbf{R}^{n}$, $n \geq 2$ and the the exact value of the $n, 1$ relative capacity of a point relative to all its bounded and open neighborhoods from $\mathbf{R}^{n}$. This value has been obtained in Theorem 6.2 (ii).

Theorem 6.4. Let $\Omega \subset \mathbf{R}^{n}$ be an open set, where $n \geq 2$ is an integer. If $u \in H_{0}^{1,(n, 1)}(\Omega)$, then $u$ has a version $u^{*} \in C(\bar{\Omega}) \cap L^{\infty}(\Omega)$ and

$$
\|u\|_{L^{\infty}(\Omega)} \leq \frac{1}{n \Omega_{n}^{1 / n}}\|\nabla u\|_{L^{n, 1}\left(\Omega ; \mathbf{R}^{n}\right)} .
$$

Moreover, if $\Omega \neq \mathbf{R}^{n}$, then $u^{*}=0$ on $\partial \Omega$.

Proof. First we prove (3) for the functions in $C_{0}^{\infty}(\Omega)$. So let $u$ be a function in $C_{0}^{\infty}(\Omega)$. We can assume without loss of generality that $u$ is not identically zero. Let $U \subset \subset \Omega$ be a bounded open set such that supp $u \subset \subset U$.

Fix $\lambda \in(0,1)$. Let $O_{\lambda}=\left\{x \in \Omega:|u(x)|>\lambda\|u\|_{L^{\infty}(\Omega)}\right\}$. Then $O_{\lambda} \subset \subset U$ is a bounded nonempty open set and the Lipschitz function $\frac{|u|}{\lambda\|u\|_{L^{\infty}(\Omega)}}$ is supported in $U$ and is admissible for the condenser $\left(O_{\lambda}, U\right)$ with respect to the $n, 1$-capacity. Let $x \in O_{\lambda}$. The monotonicity of the $\operatorname{cap}_{n, 1}(\cdot, U)$ set function and Theorem 6.2 (ii) imply

$$
n \Omega_{n}^{1 / n}=\operatorname{cap}_{n, 1}(\{x\}, U)^{1 / n} \leq \operatorname{cap}_{n, 1}\left(O_{\lambda}, U\right)^{1 / n} \leq \frac{\|\nabla u\|_{L^{n, 1}\left(\Omega ; \mathbf{R}^{n}\right)}}{\lambda\|u\|_{L^{\infty}(\Omega)}} .
$$

Thus,

$$
\|u\|_{L^{\infty}(\Omega)} \leq \frac{1}{n \Omega_{n}^{1 / n}} \cdot \lambda^{-1}\|\nabla u\|_{L^{n, 1}\left(\Omega ; \mathbf{R}^{n}\right)}
$$

for every $\lambda \in(0,1)$. By letting $\lambda \rightarrow 1$, we obtain the desired inequality for $u \in$ $C_{0}^{\infty}(\Omega)$.

Suppose now that $u \in H_{0}^{1,(n, 1)}(\Omega)$. Let $\left(u_{k}\right)_{k \geq 1} \subset C_{0}^{\infty}(\Omega)$ be a sequence that converges to $u$ in $H_{0}^{1,(n, 1)}(\Omega)$. We can assume without loss of generality that the sequence $\left(u_{k}\right)_{k \geq 1} \subset C_{0}^{\infty}(\Omega)$ is chosen such that $u_{k}$ converges pointwise to $u$ almost everywhere in $\Omega, \nabla u_{k}$ converges pointwise to $\nabla u$ almost everywhere in $\Omega$, and such that

$$
\left\|u_{k+1}-u_{k}\right\|_{L^{n, 1}(\Omega)}+\left\|\nabla u_{k+1}-\nabla u_{k}\right\|_{L^{n, 1}\left(\Omega ; \mathbf{R}^{n}\right)}<2^{-2 k}, \forall k \geq 1 .
$$

By applying the inequality (3) to the smooth functions $u_{k}$ and $u_{k+1}-u_{k}$ that are compactly supported in $\Omega$ for every $k \geq 1$, we obtain

$$
\begin{gathered}
\left|u_{k}(x)\right| \leq \frac{1}{n \Omega_{n}^{1 / n}}\left\|\nabla u_{k}\right\|_{L^{n, 1}\left(\Omega ; \mathbf{R}^{n}\right)} \quad \text { and } \\
\left|u_{k+1}(x)-u_{k}(x)\right| \leq \frac{1}{n \Omega_{n}^{1 / n}}\left\|\nabla u_{k+1}-\nabla u_{k}\right\|_{L^{n, 1}\left(\Omega ; \mathbf{R}^{n}\right)}
\end{gathered}
$$

for all $x$ in $\Omega$ and for every $k \geq 1$. From the choice of the sequence $u_{k}$ and (5), it follows that the sequence $u_{k}$ is uniformly fundamental on $\Omega$. Thus, $u_{k}$ converges uniformly in $\Omega$ to a function $v \in C(\Omega)$. Since the functions $u_{k}$ are in $C_{0}^{\infty}(\Omega)$, we can assume without loss of generality that they are in $C(\bar{\Omega})$. This is trivial when $\Omega=\mathbf{R}^{n}$; when $\Omega \neq \mathbf{R}^{n}$ (that is, when $\partial \Omega \neq \emptyset$ ), we set all the functions $u_{k}$ to be 0 
on $\partial \Omega$. Thus, the sequence $u_{k}$ is uniformly fundamental in $\bar{\Omega}$ and its uniform limit $v$ is continuous on $\bar{\Omega}$. Moreover, if $\Omega \neq \mathbf{R}^{n}$, then $v$ is 0 on $\partial \Omega$.

Since $u_{k}$ converges pointwise almost everywhere to $u$ in $\Omega$ and uniformly to $v$ in $\Omega$, it follows that $u=v$ almost everywhere in $\Omega$. Consequently, $v \in H_{0}^{1,(n, 1)}(\Omega)$ and $\nabla u=\nabla v$ almost everywhere in $\Omega$.

By letting $k \rightarrow \infty$ in (4), we obtain

$$
\begin{aligned}
|v(x)| & =\lim _{k \rightarrow \infty}\left|u_{k}(x)\right| \leq \frac{1}{n \Omega_{n}^{1 / n}} \lim _{k \rightarrow \infty}\left\|\nabla u_{k}\right\|_{L^{n, 1}\left(\Omega ; \mathbf{R}^{n}\right)} \\
& =\frac{1}{n \Omega_{n}^{1 / n}}\|\nabla u\|_{L^{n, 1}\left(\Omega ; \mathbf{R}^{n}\right)}=\frac{1}{n \Omega_{n}^{1 / n}}\|\nabla v\|_{L^{n, 1}\left(\Omega ; \mathbf{R}^{n}\right)}
\end{aligned}
$$

for every $x$ in $\Omega$. Since $u=v$ almost everywhere in $\Omega$, this implies that $u \in L^{\infty}(\Omega)$ and (3) holds. This finishes the proof of the theorem.

Remark 6.5. Suppose $n \geq 2$ is an integer. We can see easily that we have equality in (3) for the functions $u_{r}$ that were used in the proof of Theorem 6.3. In the aforementioned theorem, for a fixed $r$ in $(0, \infty)$ the function $u_{r}: \mathbf{R}^{n} \rightarrow[0,1]$ was defined by

$$
u_{r}(x)= \begin{cases}1-\frac{|x|}{r} & \text { if } 0 \leq|x| \leq r \\ 0 & \text { if }|x|>r\end{cases}
$$

As a consequence of Theorem 6.4, we now show that every function in $H_{\text {loc }}^{1,(n, 1)}(\Omega)$ has a version that is continuous on $\Omega$. It is pretty clear to see that this result also follows as a consequence of the aforementioned results obtained by Stein in [17] and by Cianchi-Pick in [2].

Proposition 6.6. Let $\Omega \subset \mathbf{R}^{n}$ be an open set, where $n \geq 2$ is an integer. Suppose that $u \in H_{\mathrm{loc}}^{1,(n, 1)}(\Omega)$. Then $u$ has a version $u^{*} \in C(\Omega)$.

Proof. Choose open sets $\emptyset=\Omega_{0} \subsetneq \Omega_{j} \subset \subset \Omega_{j+1}, j \geq 1$ such that $\bigcup_{j} \Omega_{j}=\Omega$. Like in the proof of [7, Theorem 4.11] (see also Heinonen-Kilpelainen-Martio [12, Lemma 1.15]), we construct a sequence $\psi_{j}, j \geq 1$ such that $\psi_{j} \in C_{0}^{\infty}\left(\Omega_{j+1} \backslash \bar{\Omega}_{j-1}\right)$ for every $j \geq 1$ and $\sum_{j} \psi_{j} \equiv 1$ on $\Omega$.

We notice via Lemma 4.1 that $u \psi_{j} \in H_{0}^{1,(n, 1)}(\Omega)$ is compactly supported in $\Omega$ for all $j \geq 1$. By applying Theorem 6.4 to the sequence $\left(u \psi_{j}\right)_{j \geq 1}$, we find a continuous version $\left(u \psi_{j}\right)^{*}$ of $u \psi_{j}$ that is compactly supported in $\Omega$ for every $j \geq 1$. Then $u^{*}:=\sum_{j}\left(u \psi_{j}\right)^{*}$ is a version of $u=\sum_{j} u \psi_{j}$. Since on every bounded open set $U \subset \subset \Omega$ only finitely many of the functions $\left(u \psi_{j}\right)^{*}$ are non-vanishing, it follows immediately that $u^{*}$ is in fact continuous on $\Omega$. This finishes the proof.

\section{Bounded sequences in non-reflexive Sobolev-Lorentz spaces}

Whenever we proved a Monotone Convergence Theorem for the relative and global Sobolev-Lorentz $(p, q)$ capacities associated to reflexive spaces $H_{0}^{1,(p, q)}(\Omega), 1<$ $q<\infty$, we always used the fact (via Theorems 4.3-4.4) that every bounded sequence $\left(u_{k}\right)_{k \geq 1} \subset H_{0}^{1,(p, q)}(\Omega)$ has a subsequence $\left(u_{k_{i}}\right)$ that converges weakly in $H^{1,(p, q)}(\Omega)$ to a function $u \in H_{0}^{1,(p, q)}(\Omega)$.

We know that the spaces $H^{1,(p, 1)}(\Omega)$ and $H_{0}^{1,(p, 1)}(\Omega)$ are not reflexive. See for instance the discussion from Section 4.1 in our paper [7]. In this section we prove 
a weak convergence theorem concerning $H^{1,(p, 1)}(\Omega)$ and $H_{0}^{1,(p, q)}(\Omega)$ that is similar to Theorem 4.4. The weak convergence result concerning $H^{1,(p, 1)}(\Omega)$ is valid whenever $1<p<\infty$. See Theorem 7.1 (i). The weak convergence result concerning $H_{0}^{1,(p, 1)}(\Omega)$ is valid whenever $1 \leq n<p<\infty$ or $1<n=p<\infty$. See Theorem 7.1 (ii).

This theorem shows that if a bounded sequence from $H^{1,(p, 1)}(\Omega)$ converges weakly in a reflexive space $H^{1,(p, q)}(\Omega)$ to a function from $H^{1,(p, q)}(\Omega)$ for some fixed $q$ in $(1, \infty)$, then that limiting function is in $H^{1,(p, 1)}(\Omega)$ and the sequence converges weakly to the same function in every reflexive space $H^{1,(p, s)}(\Omega), 1<s<\infty$. However, this type of convergence appears to yield less than the classical weak convergence in $L^{p, 1}(\Omega) \times L^{p, 1}\left(\Omega ; \mathbf{R}^{n}\right)$. We plan to investigate the relation between these types of convergence in a subsequent paper.

Theorem 7.1. Let $\Omega \subset \mathbf{R}^{n}$ be an open set, where $n \geq 1$ is an integer. Suppose that $1<p, q<\infty$. Let $u$ be a function in $H^{1,(p, q)}(\Omega)$ and let $\left(u_{k}\right)_{k \geq 1} \subset H^{1,(p, 1)}(\Omega)$ be a sequence that is bounded in $H^{1,(p, 1)}(\Omega)$. Suppose that $u_{k}$ converges to $u$ weakly in $L^{(p, q)}(\Omega)$ and that $\nabla u_{k}$ converges to $\nabla u$ weakly in $L^{(p, q)}\left(\Omega ; \mathbf{R}^{n}\right)$.

(i) We have that $u$ is in $H^{1,(p, 1)}(\Omega)$. Moreover, the sequence $u_{k}$ converges to $u$ weakly in $L^{(p, s)}(\Omega)$, while the sequence $\nabla u_{k}$ converges to $\nabla u$ weakly in $L^{(p, s)}\left(\Omega ; \mathbf{R}^{n}\right)$ for every $1<s<\infty$. Also, we have

$$
\begin{aligned}
\int_{\Omega} u_{k}(x) \varphi(x) d x & \rightarrow \int_{\Omega} u(x) \varphi(x) d x \quad \text { and } \\
\int_{\Omega} \partial_{i} u_{k}(x) \varphi(x) d x & \rightarrow \int_{\Omega} \partial_{i} u(x) \varphi(x) d x, \quad i=1, \ldots, n
\end{aligned}
$$

for every simple function $\varphi \in L^{p^{\prime}, \infty}(\Omega)$. Furthermore, we have

$$
\begin{aligned}
\|u\|_{H^{1,(p, 1)}(\Omega)} & \leq \liminf _{k \rightarrow \infty}\left\|u_{k}\right\|_{H^{1,(p, 1)}(\Omega)}, \\
\|u\|_{H^{1, p, 1}(\Omega)} & \leq \liminf _{k \rightarrow \infty}\left\|u_{k}\right\|_{H^{1, p, 1}(\Omega)}, \\
\|\nabla u\|_{L^{(p, 1)}\left(\Omega ; \mathbf{R}^{n}\right)} & \leq \liminf _{k \rightarrow \infty}\left\|\nabla u_{k}\right\|_{L^{(p, 1)}\left(\Omega ; \mathbf{R}^{n}\right)} \text { and } \\
\|\nabla u\|_{L^{p, 1}\left(\Omega ; \mathbf{R}^{n}\right)} & \leq \liminf _{k \rightarrow \infty}\left\|\nabla u_{k}\right\|_{L^{p, 1}\left(\Omega ; \mathbf{R}^{n}\right)} .
\end{aligned}
$$

(ii) Suppose that $1 \leq n<p<\infty$ or $1<n=p<\infty$. If $u_{j} \in H_{0}^{1,(p, 1)}(\Omega)$ for all $j \geq 1$, then $u \in H_{0}^{1,(p, 1)}(\Omega)$.

Proof. (i) We recall that $H^{1,(p, r)}(\Omega) \subset H^{1,(p, s)}(\Omega)$ and $H_{0}^{1,(p, r)}(\Omega) \subset H_{0}^{1,(p, s)}(\Omega)$ whenever $1 \leq r<s \leq \infty$. (See [7, Theorem 4.3]). Moreover, from Remark 3.2, (2) and the definition of the Sobolev-Lorentz spaces and norms on $\Omega$, it follows that there exists a constant $C(p, r, s)>0$ such that

$$
\|v\|_{H^{1,(p, s)}(\Omega)} \leq C(p, r, s)\|v\|_{H^{1,(p, r)}(\Omega)}
$$

for every $v \in H^{1,(p, r)}(\Omega)$. Thus, any sequence that is bounded in $H^{1,(p, 1)}(\Omega)$ is also bounded in $H^{1,(p, s)}(\Omega)$ whenever $1<s<\infty$. Similarly, any sequence that is bounded in $H_{0}^{1,(p, 1)}(\Omega)$ is also bounded in $H_{0}^{1,(p, s)}(\Omega)$ whenever $1<s<\infty$. The spaces $H^{1,(p, s)}(\Omega)$ and $H_{0}^{1,(p, s)}(\Omega)$ are reflexive whenever $1<p, s<\infty$. See the discussion before Theorem 4.1 from our paper [7].

Let $q$ in $(1, \infty)$ be fixed. From Theorem 4.4 it follows that for any sequence $\widetilde{u}_{k}$ that is bounded in $H^{1,(p, q)}(\Omega)$ there is a subsequence $\widetilde{u}_{k_{i}}$ and a function $\widetilde{u} \in H^{1,(p, q)}(\Omega)$ such that $\widetilde{u}_{k_{i}}$ converges weakly to $\widetilde{u}$ in $L^{(p, q)}(\Omega)$ and $\nabla \widetilde{u}_{k_{i}}$ converges weakly to $\nabla \widetilde{u}$ in 
$L^{(p, q)}\left(\Omega ; \mathbf{R}^{n}\right)$. Moreover, from Theorem 4.4 it follows that $\widetilde{u}$ is in $H_{0}^{1,(p, q)}(\Omega)$ if all the functions $\widetilde{u}_{j}$ are in $H_{0}^{1,(p, q)}(\Omega)$.

If such a sequence $\widetilde{u}_{k}$ is bounded in $H^{1,(p, 1)}(\Omega)$, from (11), Theorem 4.4 and from the discussion in the previous paragraph we obtain the existence of a subsequence $\widetilde{u}_{k_{i}}$ and of a function $\widetilde{u} \in H^{1,(p, q)}(\Omega)$ such that $\widetilde{u}_{k_{i}}$ converges weakly in $L^{p, q}(\Omega)$ to $\widetilde{u}$ and such that $\nabla \widetilde{u}_{k_{i}}$ converges weakly to $\nabla \widetilde{u}$ in $L^{p, q}\left(\Omega ; \mathbf{R}^{n}\right)$. Moreover, from the discussion in the previous paragraph and from (11) it follows that $\widetilde{u}$ is in $H_{0}^{1,(p, q)}(\Omega)$ if all the functions $\widetilde{u}_{j}$ are in $H_{0}^{1,(p, 1)}(\Omega)$.

However, since the subsequence $\widetilde{u}_{k_{i}}$ is bounded in the non-reflexive space $H^{1,(p, 1)}$ $(\Omega)$, it is also bounded via $(11)$ in the reflexive spaces $H^{1,(p, s)}(\Omega)$ for every $1<$ $s<\infty$. From this, the fact that $\widetilde{u}$ is the weak limit of the subsequence $\widetilde{u}_{k_{i}}$ in $H^{1,(p, q)}(\Omega)$, from the definition of the spaces $L^{p, s}\left(\Omega ; \mathbf{R}^{m}\right)$, from Hunt [13, p. 258] and from Bennett-Sharpley [1, Theorem IV.4.7 and Corollary IV.4.8], it follows in fact that $\widetilde{u} \in H^{1,(p, s)}(\Omega)$ for every $1<s<\infty$. Moreover, $\widetilde{u}_{k_{i}}$ converges weakly in $L^{p, s}(\Omega)$ to $\widetilde{u}$ and $\nabla \widetilde{u}_{k_{i}}$ converges weakly to $\nabla \widetilde{u}$ in $L^{p, s}\left(\Omega ; \mathbf{R}^{n}\right)$ for every $1<s<\infty$.

Thus, if we have a bounded sequence $u_{k}$ in $H^{1,(p, 1)}(\Omega)$ that converges weakly in $H^{1,(p, q)}(\Omega)$ to a function $u \in H^{1,(p, q)}(\Omega)$, the above argument shows that $u$ belongs to $H^{1,(p, s)}(\Omega), u_{k}$ converges weakly in $L^{p, s}(\Omega)$ to $u$ and $\nabla u_{k}$ converges weakly to $\nabla u$ in $L^{p, s}\left(\Omega ; \mathbf{R}^{n}\right)$ for every $1<s<\infty$. Moreover, (6) holds for $u$ and for the sequence $u_{k}$. If in addition the sequence $\left(u_{k}\right)_{k \geq 1} \subset H_{0}^{1,(p, 1)}(\Omega)$ is bounded in $H_{0}^{1,(p, 1)}(\Omega)$, from the previous argument and Theorem 4.4 it follows that the function $u$ is in fact in $H_{0}^{1,(p, s)}(\Omega)$ for all $1<s<\infty$.

From (6) it follows easily via Fatou's Lemma and via the Hölder inequality for Lorentz spaces (see [5, Theorem 2.3] and/or [7, Theorem 3.7]) that

$$
\begin{aligned}
\left|\int_{\Omega} u(x) \varphi(x) d x\right| & \leq\left(\liminf _{k \rightarrow \infty}\left\|u_{k}\right\|_{L^{p, 1}(\Omega)}\right)\|\varphi\|_{L^{p^{\prime}, \infty}(\Omega)} \quad \text { and } \\
\left|\int_{\Omega} \partial_{i} u(x) \varphi(x) d x\right| & \leq\left(\liminf _{k \rightarrow \infty}\left\|\partial_{i} u_{k}\right\|_{L^{p, 1}(\Omega)}\right)\|\varphi\|_{L^{p^{\prime}, \infty}(\Omega)}, \quad i=1, \ldots, n,
\end{aligned}
$$

for every simple function $\varphi \in L^{p^{\prime}, \infty}(\Omega)$.

From (12) and Bennett-Sharpley [1, Proposition I.3.13, Theorems I.4.1 and IV.4.7] it follows that $u$ is in $L^{p, 1}(\Omega)$. From (13) and Bennett-Sharpley [1, Proposition I.3.13, Theorems I.4.1 and IV.4.7] it follows that $\partial_{i} u$ is in $L^{p, 1}(\Omega)$ for $i=\overline{1, n}$. Thus, $u$ is in $W^{1,(p, 1)}(\Omega)$. Since $W^{1,(p, 1)}(\Omega)=H^{1,(p, 1)}(\Omega)$ (see [7, Theorem 4.11]), it follows that $u$ is indeed in $H^{1,(p, 1)}(\Omega)$.

Thus, we finally showed that $u$ is in $H^{1,(p, 1)}(\Omega)$. Now we prove that

$$
\begin{aligned}
\|u\|_{L^{(p, 1)}(\Omega)} & \leq \liminf _{k \rightarrow \infty}\left\|u_{k}\right\|_{L^{(p, 1)}(\Omega)} \quad \text { and } \\
\left\|\partial_{i} u\right\|_{L^{(p, 1)}(\Omega)} & \leq \liminf _{k \rightarrow \infty}\left\|\partial_{i} u_{k}\right\|_{L^{(p, 1)}(\Omega)}, \quad i=1, \ldots, n .
\end{aligned}
$$

From (6) and Stein-Weiss [18, Lemma V.3.17 (i) and (iii)] it follows that whenever $0<t \leq|\Omega|$ there exist Lebesgue measurable sets $E_{t}$ and $E_{t, i} \subset \Omega, i=1, \ldots n$ such 
that $\left|E_{t}\right|=\left|E_{t, i}\right|=t, i=1, \ldots, n$ and such that

$$
\begin{aligned}
u^{* *}(t) & =\frac{1}{t} \int_{0}^{t} u^{*}(s) d s=\frac{1}{t} \int_{E_{t}}|u(x)| d x \leq \liminf _{k \rightarrow \infty} \frac{1}{t} \int_{E_{t}}\left|u_{k}(x)\right| d x \\
& \leq \liminf _{k \rightarrow \infty} \frac{1}{t} \int_{0}^{t}\left|u_{k}\right|^{*}(s) d s=\liminf _{k \rightarrow \infty} u_{k}^{* *}(t) \text { and } \\
\left|\partial_{i} u\right|^{* *}(t) & =\frac{1}{t} \int_{0}^{t}\left|\partial_{i} u\right|^{*}(s) d s=\frac{1}{t} \int_{E_{t, i}}\left|\partial_{i} u(x)\right| d x \leq \liminf _{k \rightarrow \infty} \frac{1}{t} \int_{E_{t, i}}\left|\partial_{i} u_{k}(x)\right| d x \\
& \leq \liminf _{k \rightarrow \infty} \frac{1}{t} \int_{0}^{t}\left|\partial_{i} u_{k}\right|^{*}(s) d s=\liminf _{k \rightarrow \infty}\left|\partial_{i} u_{k}\right|^{* *}(t), \quad i=1, \ldots, n .
\end{aligned}
$$

Now (14) follows from (16) and Fatou's Lemma, while (15) follows from (17) and Fatou's Lemma.

Since we do not know whether the sequence $\left(u_{k}, \nabla u_{k}\right)$ converges weakly to $(u, \nabla u)$ in $L^{p, 1}(\Omega) \times L^{p, 1}\left(\Omega ; \mathbf{R}^{n}\right)$ or not, we cannot use the weak- ${ }^{\star}$ lower semicontinuity of the $L^{p, 1}$ quasinorm in order to derive (7)-(10). Thus, we have to use a different approach in order to obtain (7)-(10).

We can choose subsequences $u_{k, 1}, u_{k, 2}, u_{k, 3}$ and $u_{k, 4}$ such that

$$
\begin{aligned}
\lim _{k \rightarrow \infty}\left\|u_{k, 1}\right\|_{H^{1,(p, 1)}(\Omega)} & =\liminf _{k \rightarrow \infty}\left\|u_{k}\right\|_{H^{1,(p, 1)}(\Omega)}, \\
\lim _{k \rightarrow \infty}\left\|u_{k, 2}\right\|_{H^{1, p, 1}(\Omega)} & =\liminf _{k \rightarrow \infty}\left\|u_{k}\right\|_{H^{1, p, 1}(\Omega)}, \\
\lim _{k \rightarrow \infty}\left\|\nabla u_{k, 3}\right\|_{L^{(p, 1)}\left(\Omega ; \mathbf{R}^{n}\right)} & =\liminf _{k \rightarrow \infty}\left\|\nabla u_{k}\right\|_{L^{(p, 1)}\left(\Omega ; \mathbf{R}^{n}\right)} \quad \text { and } \\
\lim _{k \rightarrow \infty}\left\|\nabla u_{k, 4}\right\|_{L^{p, 1}\left(\Omega ; \mathbf{R}^{n}\right)} & =\liminf _{k \rightarrow \infty}\left\|\nabla u_{k}\right\|_{L^{p, 1}\left(\Omega ; \mathbf{R}^{n}\right)} .
\end{aligned}
$$

We can apply the Mazur lemma to the sequences $\left(u_{k, i}, \nabla u_{k, i}\right), i=1, \ldots, 4$, with respect to the reflexive space $L^{(p, q)}(\Omega) \times L^{(p, q)}\left(\Omega ; \mathbf{R}^{n}\right)$ to obtain sequences $v_{k, i}$ of convex combinations of $u_{k, i}, i=1, \ldots, 4$ such that $v_{k, i} \rightarrow u$ in $H^{1,(p, q)}(\Omega), v_{k, i} \rightarrow u$ almost everywhere in $\Omega$ and $\nabla v_{k, i} \rightarrow \nabla u$ almost everywhere in $\Omega, i=\overline{1,4}$.

We present here the construction argument for the Mazur lemma. Let $k_{0} \geq 1$ and $1 \leq i \leq 4$ be fixed. Since every subsequence of $\left(u_{k, i}, \nabla u_{k, i}\right)$ converges to $(u, \nabla u)$ weakly in $L^{(p, q)}(\Omega) \times L^{(p, q)}\left(\Omega ; \mathbf{R}^{n}\right)$, we may use the Mazur lemma for the subsequence $u_{k, i}, k \geq k_{0}$ with respect to $L^{(p, q)}(\Omega) \times L^{(p, q)}\left(\Omega ; \mathbf{R}^{n}\right)$. We obtain a finite convex combination $v_{k_{0}, i}$ of the functions $u_{k, i}, k \geq k_{0}$,

$$
v_{k_{0}, i}=\sum_{j=k_{0}}^{j_{k_{0}, i}} \lambda_{k_{0}, j, i} u_{j, i}, \quad \lambda_{k_{0}, j, i} \geq 0 \quad \text { and } \quad \sum_{j=k_{0}}^{j_{k_{0}, i}} \lambda_{k_{0}, j, i}=1
$$

as close to $u$ as we want in $H^{1,(p, q)}(\Omega)$ (but not necessarily in $H^{1,(p, 1)}(\Omega)$ ).

A finite convex combination of functions from $H^{1,(p, 1)}(\Omega)$ is a function from $H^{1,(p, 1)}(\Omega)$. Moreover, for every $i=1, \ldots, 4$ we have $\left(v_{k, i}\right)_{k \geq 1} \subset H_{0}^{1,(p, 1)}(\Omega)$ if $\left(u_{k}\right)_{k \geq 1} \subset H_{0}^{1,(p, 1)}(\Omega)$. Passing to subsequences if necessary, we may assume for every $i=1, \ldots, 4$ that $v_{k, i} \rightarrow u$ almost everywhere in $\Omega, \nabla v_{k, i} \rightarrow \nabla u$ almost everywhere in $\Omega$ and that

$$
\left\|v_{k+1, i}-v_{k, i}\right\|_{L^{(p, q)}(\Omega)}+\left\|\nabla v_{k+1, i}-\nabla v_{k, i}\right\|_{L^{(p, q)}\left(\Omega ; \mathbf{R}^{n}\right)}<2^{-2 k}
$$

for every $k \geq 1$.

This ends the construction of the sequences $\left(v_{k, i}\right)_{k>1} \subset H^{1,(p, 1)}(\Omega), i=1, \ldots, 4$ by using the Mazur lemma with respect to $L^{(p, q)}(\Omega) \times L^{(p, q)}\left(\Omega ; \mathbf{R}^{n}\right)$. 
Now we finish the prove of claim (i). From the convexity of both the $p, 1$-norm and the $(p, 1)$-norm, the choice of the sequences $u_{k, i}$, and the definition of the functions $v_{k, i}, i=1, \ldots, 4$ we obtain

$$
\begin{aligned}
\liminf _{k \rightarrow \infty}\left\|v_{k, 1}\right\|_{H^{1,(p, 1)}(\Omega)} & \leq \liminf _{k \rightarrow \infty}\left\|u_{k}\right\|_{H^{1,(p, 1)}(\Omega)}, \\
\liminf _{k \rightarrow \infty}\left\|v_{k, 2}\right\|_{H^{1, p, 1}(\Omega)} & \leq \liminf _{k \rightarrow \infty}\left\|u_{k}\right\|_{H^{1, p, 1}(\Omega)}, \\
\liminf _{k \rightarrow \infty}\left\|\nabla v_{k, 3}\right\|_{L^{(p, 1)}\left(\Omega ; \mathbf{R}^{n}\right)} & \leq \liminf _{k \rightarrow \infty}\left\|\nabla u_{k}\right\|_{L^{(p, 1)}\left(\Omega ; \mathbf{R}^{n}\right)} \quad \text { and } \\
\liminf _{k \rightarrow \infty}\left\|\nabla v_{k, 4}\right\|_{L^{p, 1}\left(\Omega ; \mathbf{R}^{n}\right)} & \leq \liminf _{k \rightarrow \infty}\left\|\nabla u_{k}\right\|_{L^{p, 1}\left(\Omega ; \mathbf{R}^{n}\right) .}
\end{aligned}
$$

Since the sequences $v_{k, i}, k \geq 1$ converge pointwise almost everywhere on $\Omega$ to $u$ for every $i=1, \ldots, 4$, it follows via Bennett-Sharpley [1, Proposition II.1.7] and via Fatou's Lemma that for every $i=1, \ldots, 4$ we have

$$
u^{*}(t) \leq \liminf _{k \rightarrow \infty} v_{k, i}^{*}(t) \text { and } u^{* *}(t) \leq \liminf _{k \rightarrow \infty} v_{k, i}^{* *}(t) \text { for every } t>0 .
$$

Similarly, since the sequences $\nabla v_{k, i}, k \geq 1$ converge pointwise almost everywhere on $\Omega$ to $\nabla u$ for every $i=1, \ldots, 4$, it follows via Bennett-Sharpley [1, Proposition II.1.7] and via Fatou's Lemma that for every $i=1, \ldots, 4$ we have

$$
|\nabla u|^{*}(t) \leq \liminf _{k \rightarrow \infty}\left|\nabla v_{k, i}\right|^{*}(t) \quad \text { and } \quad|\nabla u|^{* *}(t) \leq \liminf _{k \rightarrow \infty}\left|\nabla v_{k, i}\right|^{* *}(t)
$$

for every $t>0$. Moreover, from (22), (23), (24) and (25) we have via (26) and (27) and via Fatou's Lemma

$$
\begin{aligned}
\|u\|_{H^{1,(p, 1)}(\Omega)} & \leq \liminf _{k \rightarrow \infty}\left\|v_{k, 1}\right\|_{H^{1,(p, 1)}(\Omega)} \leq \liminf _{k \rightarrow \infty}\left\|u_{k}\right\|_{H^{1,(p, 1)}(\Omega)}, \\
\|u\|_{H^{1, p, 1}(\Omega)} & \leq \liminf _{k \rightarrow \infty}\left\|v_{k, 2}\right\|_{H^{1, p, 1}(\Omega)} \leq \liminf _{k \rightarrow \infty}\left\|u_{k}\right\|_{H^{1, p, 1}(\Omega)}, \\
\|\nabla u\|_{L^{(p, 1)}\left(\Omega ; \mathbf{R}^{n}\right)} & \leq \liminf _{k \rightarrow \infty}\left\|\nabla v_{k, 3}\right\|_{L^{(p, 1)}\left(\Omega ; \mathbf{R}^{n}\right)} \leq \liminf _{k \rightarrow \infty}\left\|\nabla u_{k}\right\|_{L^{(p, 1)}\left(\Omega ; \mathbf{R}^{n}\right)} \quad \text { and } \\
\|\nabla u\|_{L^{p, 1}\left(\Omega ; \mathbf{R}^{n}\right)} & \leq \liminf _{k \rightarrow \infty}\left\|\nabla v_{k, 4}\right\|_{L^{p, 1}\left(\Omega ; \mathbf{R}^{n}\right)} \leq \liminf _{k \rightarrow \infty}\left\|\nabla u_{k}\right\|_{L^{p, 1}\left(\Omega ; \mathbf{R}^{n}\right)} .
\end{aligned}
$$

This finishes the proof of claim (i).

(ii) Now we prove the second claim of the theorem. Before we start the proof of claim (ii), we recall that in part (i) we proved that if we have a sequence $\left(u_{k}\right)_{k \geq 1} \subset$ $H_{0}^{1,(p, 1)}(\Omega)$ that is bounded in $H^{1,(p, 1)}(\Omega)$ such that $u_{k}$ converges to $u$ weakly in $L^{(p, q)}(\Omega)$ and such that $\nabla u_{k}$ converges to $\nabla u$ weakly in $L^{(p, q)}\left(\Omega ; \mathbf{R}^{n}\right)$ for some $q$ in $(1, \infty)$, then $u$ in $H_{0}^{1,(p, s)}(\Omega)$ whenever $1<s<\infty$. Moreover, we also proved in part (i) that the sequence $u_{k}$ converges to $u$ weakly in $L^{(p, s)}(\Omega)$ and that the sequence $\nabla u_{k}$ converges to $\nabla u$ weakly in $L^{(p, s)}\left(\Omega ; \mathbf{R}^{n}\right)$ whenever $1<s<\infty$. Furthermore, we also proved that $u$ is in $H^{1,(p, 1)}(\Omega)$. The result in part (i) is valid whenever $1<p<\infty$.

Now we show that if the sequence $\left(u_{k}\right)_{k \geq 1} \subset H_{0}^{1,(p, 1)}(\Omega)$ is bounded in $H^{1,(p, 1)}(\Omega)$, $u_{k}$ converges to $u$ weakly in $L^{(p, q)}(\Omega)$ and $\nabla u_{k}$ converges to $\nabla u$ weakly in $L^{(p, q)}\left(\Omega ; \mathbf{R}^{n}\right)$ for some $q$ in $(1, \infty)$, then $u \in H_{0}^{1,(p, 1)}(\Omega)$ provided that $1 \leq n<p<\infty$ or $1<n=$ $p<\infty$.

Under the hypotheses of claim (ii) we can assume without loss of generality via the discussion at the beginning of the proof of this theorem together with our previous results [7, Theorem 5.5 (iii)] when $1=n<p<\infty$ and respectively [7, Theorem 5.6 (iv)] when $1<n<p<\infty$ that $u$ and all the functions $u_{k}$ and $v_{k, i}, k \geq 1, i=1, \ldots, 4$ are Hölder continuous with exponent $1-\frac{n}{p}$ on the closed set $\bar{\Omega}$. Moreover, if $\partial \Omega \neq \emptyset$, all these functions are 0 on $\partial \Omega$. Furthermore, from the construction of the sequences 
$v_{k, i}$, from our previous results [7, Theorem 5.5 (iii)] when $1=n<p<\infty$ and respectively [7, Theorem 5.6 (iv)] when $1<n<p<\infty$, it follows that the sequences $v_{k, i}$ converge uniformly to $u$ on compact subsets of $\Omega$ (or respectively uniformly on $\bar{\Omega}$ if $\Omega$ is bounded).

When $1<n=p<\infty$, we can assume without loss of generality via Theorem 6.6 that $u$ is continuous on $\Omega$. Moreover, when $1<n=p<\infty$ we can also assume without loss of generality via Theorem 6.4 that all the functions $u_{k}$ and $v_{k, i}$ are continuous on $\bar{\Omega}$ and in addition, if $\partial \Omega \neq \emptyset$, all of them are 0 on $\partial \Omega$.

We notice that claim (ii) holds trivially for all $p$ in $(1, \infty)$ when $\Omega=\mathbf{R}^{n}$ via our previous result [7, Theorem 4.12] or when $u$ is compactly supported in $\Omega$ via our previous result [7, Lemma 4.21].

We need to prove claim (ii) when $\Omega \neq \mathbf{R}^{n}$. We have to consider two separate cases, $\Omega$ bounded and $\Omega \neq \mathbf{R}^{n}$ unbounded. Before we differentiate between the cases $\Omega$ bounded and $\Omega \neq \mathbf{R}^{n}$ unbounded, we extend the functions $u_{k}, v_{k, i}$ and $u$ by zero on the nonempty set $\mathbf{R}^{n} \backslash \Omega$ and we denote these extensions by $\widetilde{u}_{k}, \widetilde{v}_{k, i}$ and $\widetilde{u}$ respectively. From the discussion at the beginning of the proof of claim (ii) it follows that all the functions $\widetilde{u}_{k}$ and $\widetilde{v}_{k, i}$ are continuous on $\mathbf{R}^{n}$. From our previous result $[7$, Proposition 5.2] and from the hypothesis of claim (ii), we see that the sequence $\widetilde{u}_{k}$ is bounded in $H_{0}^{1,(p, 1)}\left(\mathbf{R}^{n}\right), \widetilde{u}_{k}$ converges weakly to $\widetilde{u}$ in $L^{(p, q)}\left(\mathbf{R}^{n}\right)$ and $\nabla \widetilde{u}_{k}$ converges weakly to $\nabla \widetilde{u}$ in $L^{(p, q)}\left(\mathbf{R}^{n} ; \mathbf{R}^{n}\right)$. Thus, via claim (i) it follows that $\widetilde{u} \in H_{0}^{1,(p, 1)}\left(\mathbf{R}^{n}\right)$. This implies via [7, Theorem 5.5 (iii)] when $1=n<p<\infty$, via [7, Theorem 5.6 (iv)] when $1<n<p<\infty$ and respectively via Theorem 6.6 when $1<n=p<\infty$ that $\widetilde{u}$ has a version $\widetilde{u}^{*} \in C\left(\mathbf{R}^{n}\right)$. Since $\widetilde{u}^{*}$ is continuous on $\mathbf{R}^{n}, u$ is continuous on $\Omega$ and the restriction of $\widetilde{u}^{*}$ to $\Omega$ is a version of $u$, it follows immediately that $u=\widetilde{u}^{*}=\widetilde{u}$ everywhere in $\Omega$.

Now we consider the cases $\Omega$ bounded and $\Omega \neq \mathbf{R}^{n}$ unbounded separately.

Case 1. We start with the case when $\Omega$ is bounded. We saw already that $u=\widetilde{u}^{*}=\widetilde{u}$ everywhere in $\Omega$. Since $\widetilde{u}$ is zero everywhere on $\mathbf{R}^{n} \backslash \Omega \supset \partial \Omega$ and its version $\widetilde{u}^{*}$ is in $C_{0}\left(\mathbf{R}^{n}\right)$, it follows in fact that $\widetilde{u}^{*}$ is zero everywhere in $\mathbf{R}^{n} \backslash \Omega \supset \partial \Omega$ along with $\widetilde{u}$. Thus, $\widetilde{u}=\widetilde{u}^{*}$ everywhere on $\mathbf{R}^{n}$, both of them are zero on $\partial \Omega$ (along with $u$ ) and $\widetilde{u}^{*}=\widetilde{u}=u$ in $\Omega$. Thus, we proved that $u$ is a continuous function on $\Omega$ that extends continuously by 0 on $\partial \Omega$. This implies via our previous result $[7$, Lemma 4.21] that $u \in H_{0}^{1,(p, 1)}(\Omega)$. Thus, claim (ii) holds when $\Omega$ is bounded provided that $1 \leq n<p<\infty$ or $1<n=p<\infty$.

Case 2. We consider now the case when $\Omega \neq \mathbf{R}^{n}$ is unbounded. Without loss of generality we can assume that $0 \in \Omega \neq \mathbf{R}^{n}$. Like in the proof of [7, Theorem 4.12], we choose a sequence of 2-Lipschitz smooth functions $\left(\phi_{j}\right)_{j \geq 1} \subset C_{0}^{\infty}\left(\mathbf{R}^{n}\right)$ such that $0 \leq \phi_{j} \leq 1, \phi_{j}=1$ on $B(0, j)$ and such that $\phi_{j}$ is compactly supported in $B(0, j+1)$ for every $j \geq 1$. We recall that in the discussion before the proof of Case 1 , we extended the functions $u_{k}, v_{k, i}$ and $u$ by zero on $\mathbf{R}^{n} \backslash \Omega$ and we denoted these extensions by $\widetilde{u}_{k}, \widetilde{v}_{k, i}$ and $\widetilde{u}$ respectively. We noticed then that all the functions $\widetilde{u}_{k}$ and $\widetilde{v}_{k, i}$ are continuous on $\mathbf{R}^{n}$.

Let $j \geq 1$ be a fixed integer and let $s \geq 1$ be a finite number. Let $\Omega_{j}:=$ $\Omega \cap B(0, j+1)$. Via Lemma 4.1 and Remark 4.2 (see also [7, Lemma 4.9 and Theorem 4.11]) we have that $w \phi_{j}$ is in $H_{0}^{1,(p, s)}\left(\Omega_{j}\right)$ whenever $w$ is in $H_{0}^{1,(p, s)}(\Omega)$ with

$$
\left\|w \phi_{j}\right\|_{H^{1,(p, s)}\left(\Omega_{j}\right)} \leq 3\|w\|_{H^{1,(p, s)}(\Omega)}
$$


for all $w \in H_{0}^{1,(p, s)}(\Omega)$. Thus, via Lemma 4.1 and Remark 4.2 (see also [7, Lemma 4.9 and Theorem 4.11]), we see that the sequence $u_{k} \phi_{j}$ is bounded in $H_{0}^{1,(p, 1)}\left(\Omega_{j}\right)$ since $\phi_{j} \in C_{0}^{\infty}\left(\mathbf{R}^{n}\right)$ is compactly supported in $B(0, j+1)$ and since the sequence $u_{k}$ is bounded in $H_{0}^{1,(p, 1)}(\Omega)$.

It is also easy to see via [7, Lemma 4.9] that we have $u_{k} \phi_{j} \rightarrow u \phi_{j}$ weakly in $L^{(p, q)}\left(\Omega_{j}\right)$ and $\nabla\left(u_{k} \phi_{j}\right) \rightarrow \nabla\left(u \phi_{j}\right)$ weakly in $L^{(p, q)}\left(\Omega_{j} ; \mathbf{R}^{n}\right)$ since $\phi_{j} \in C_{0}^{\infty}\left(\mathbf{R}^{n}\right)$ is compactly supported in $B(0, j+1), u_{k} \rightarrow u$ weakly in $L^{(p, q)}(\Omega)$ and since $\nabla u_{k} \rightarrow \nabla u$ weakly in $L^{(p, q)}\left(\Omega ; \mathbf{R}^{n}\right)$. By applying Case 1 to the sequence $\left(u_{k} \phi_{j}\right)_{k \geq 1}$ with respect to the bounded open set $\Omega_{j}$, we see that $u \phi_{j} \in H_{0}^{1,(p, 1)}\left(\Omega_{j}\right)$. Thus, $u \phi_{j} \in H_{0}^{1,(p, 1)}\left(\Omega_{j}\right) \subset$ $H_{0}^{1,(p, 1)}(\Omega)$ for every $j \geq 1$ integer. By doing a computation similar to the one from the proof of our previous result [7, Theorem 4.12], we obtain

$$
\begin{aligned}
\left\|\widetilde{u}-\widetilde{u} \phi_{j}\right\|_{H^{1,(p, 1)}\left(\mathbf{R}^{n}\right) \leq} & \left\|\widetilde{u}\left(1-\phi_{j}\right)\right\|_{L^{(p, 1)}\left(\mathbf{R}^{n}\right)}+\left\|\widetilde{u} \nabla \phi_{j}\right\|_{L^{(p, 1)}\left(\mathbf{R}^{n} ; \mathbf{R}^{n}\right)} \\
& +\left\|\left(1-\phi_{j}\right) \nabla \widetilde{u}\right\|_{L^{(p, 1)}\left(\mathbf{R}^{n} ; \mathbf{R}^{n}\right)} \\
\leq & 3\left\|\widetilde{u} \chi_{\mathbf{R}^{n} \backslash B(0, j)}\right\|_{L^{(p, 1)}\left(\mathbf{R}^{n}\right)}+\left\|\nabla \widetilde{u} \chi_{\mathbf{R}^{n} \backslash B(0, j)}\right\|_{L^{(p, 1)}\left(\mathbf{R}^{n} ; \mathbf{R}^{n}\right)} \rightarrow 0
\end{aligned}
$$

as $j \rightarrow \infty$. From this, [7, Proposition 5.2], the definition of $\widetilde{u}$ and the fact that $\widetilde{u} \phi_{j}$ is the extension by 0 on $\mathbf{R}^{n} \backslash \Omega_{j}$ of $u \phi_{j} \in H_{0}^{1,(p, 1)}\left(\Omega_{j}\right) \subset H_{0}^{1,(p, 1)}(\Omega)$ for every $j \geq 1$ integer, it follows that $u \in H_{0}^{1,(p, 1)}(\Omega)$. This finishes the proof of the case $\Omega \neq \mathbf{R}^{n}$ unbounded. Thus, we finish proving claim (ii) and the theorem.

Remark 7.2. When proving this weak convergence result for $H_{0}^{1,(p, 1)}(\Omega)$, we relied heavily many times on the fact that we can work with continuous functions from $H_{0}^{1,(p, 1)}(\Omega)$ whenever $1 \leq n<p<\infty$ or $1<n=p<\infty$. The existence of discontinuous and/or unbounded functions in $H_{0}^{1,(p, 1)}(\Omega)$ when $1<p<n$ leaves as an open question the membership of the limit function $u$ in $H_{0}^{1,(p, 1)}(\Omega)$ when $1<p<n$, $\Omega \subset \mathbf{R}^{n}$ is bounded and $u$ is not compactly supported in $\Omega$. Thus, we do not know at this point in time whether the weak convergence result concerning $H_{0}^{1,(p, 1)}(\Omega)$ can be extended to the case $1<p<n$.

The following proposition will be useful in the sequel.

Proposition 7.3. Suppose that $1<n, q<\infty$, where $n$ is an integer. Let $\Omega \subset \mathbf{R}^{n}$ be an open set. Let $u$ be a function in $C(\Omega) \cap H_{0}^{1,(n, 1)}(\Omega)$ and let $\left(u_{k}\right)_{k \geq 1} \subset$ $C(\Omega) \cap H_{0}^{1,(n, 1)}(\Omega)$ be a sequence in $H_{0}^{1,(n, 1)}(\Omega)$ such that

$$
\left\|u_{k}-u\right\|_{L^{(n, q)}(\Omega)}+\left\|\nabla u_{k}-\nabla u\right\|_{L^{(n, q)}\left(\Omega ; \mathbf{R}^{n}\right)}<2^{-2 k}
$$

for every $k \geq 1$. Then there exists a Borel set $F \subset \Omega$ such that $\operatorname{Cap}_{n, q}(F)=0$ and such that $u_{k} \rightarrow u$ pointwise on $\Omega \backslash F$.

Proof. For every $k \geq 1$ let

$$
O_{k}=\left\{x \in \Omega:\left|u_{k+1}(x)-u_{k}(x)\right|>2^{-k}\right\} \quad \text { and } \quad U_{k}=\bigcup_{l \geq k} O_{l} .
$$

Since all the functions $u_{j}$ are continuous on $\Omega$, it follows that $O_{k}$ is in fact an open subset of $\Omega$ for every $k \geq 1$. For every $k \geq 1$, the function $w_{k}:=2^{k}\left|u_{k+1}-u_{k}\right|$ is 
admissible for the open set $O_{k}$ with respect to the global $(n, q)$-capacity and we have

$$
\begin{aligned}
\operatorname{Cap}_{(n, q)}\left(O_{k}\right)^{1 / n} \leq & \left\|w_{k}\right\|_{L^{(n, q)}(\Omega)}+\left\|\nabla w_{k}\right\|_{L^{(n, q)}\left(\Omega ; \mathbf{R}^{n}\right)} \\
= & 2^{k}\left(\left\|u_{k+1}-u_{k}\right\|_{L^{(n, q)}(\Omega)}+\left\|\nabla u_{k+1}-\nabla u_{k}\right\|_{L^{(n, q)}\left(\Omega ; \mathbf{R}^{n}\right)}\right) \\
\leq & 2^{k}\left(\left\|u_{k+1}-u\right\|_{L^{(n, q)}(\Omega)}+\left\|\nabla u_{k+1}-\nabla u\right\|_{L^{(n, q)}\left(\Omega ; \mathbf{R}^{n}\right)}\right) \\
& +2^{k}\left(\left\|u_{k}-u\right\|_{L^{(n, q)}(\Omega)}+\left\|\nabla u_{k}-\nabla u\right\|_{L^{(n, q)}\left(\Omega ; \mathbf{R}^{n}\right)}\right) \\
< & 2^{k}\left(2^{-2(k+1)}+2^{-2 k}\right)<2^{1-k} .
\end{aligned}
$$

The set $U_{k}$ is a countable union of open sets in $\Omega$, hence it an open set in $\Omega$ itself and

$$
\operatorname{Cap}_{n, q}\left(U_{k}\right)^{1 / n} \leq \operatorname{Cap}_{(n, q)}\left(U_{k}\right)^{1 / n} \leq \sum_{j=k}^{\infty} \operatorname{Cap}_{(n, q)}\left(O_{j}\right)^{1 / n} \leq \sum_{j=k}^{\infty} 2^{1-j}=2^{2-k}
$$

Let $F=\bigcap_{k \geq 1} U_{k}$. It follows immediately that $F$ is a Borel set and $\operatorname{Cap}_{n, q}(F)=$ $\operatorname{Cap}_{(n, q)}(F)=0$. Let $v: \Omega \rightarrow \mathbf{R}$ be the function

$$
v(x)= \begin{cases}\lim _{k \rightarrow \infty} u_{k}(x) & \text { if } x \in \Omega \backslash F \\ 0 & \text { if } x \in F\end{cases}
$$

We notice that $u_{k}$ converges to $v$ pointwise in $\Omega \backslash F$ and uniformly on the sets $\Omega \backslash U_{j}, j \geq 1$. In particular $v$ is continuous when restricted to the sets $\Omega \backslash U_{j}, j \geq 1$. We know that $u=v$ almost everywhere in $\Omega$ since the sequence $u_{k}$ converges to $u$ in $H_{0}^{1,(n, q)}(\Omega)$ and to $v$ almost everywhere in $\Omega$. We claim that $u=v$ on $\Omega \backslash F$. This would imply that that $v_{k}$ converges to $u$ pointwise in $\Omega \backslash F$ and uniformly on the sets $\Omega \backslash U_{j}, j \geq 1$.

In order to prove that $u=v$ on $\Omega \backslash F$, it is enough to prove that $u=v$ on $\Omega \backslash U_{j}$ for all $j \geq 1$ since $F=\bigcap_{j \geq 1} U_{j}$. Let $j \geq 1$ be fixed. We study two separate cases here, depending on whether $\Omega$ is bounded or not.

Case 1. Assume that $\Omega$ is bounded. We can assume without loss of generality via Theorem 6.4 that $u$ and the functions $u_{k}$ are continuous on $\bar{\Omega}$ and 0 on $\partial \Omega$. We can also extend $v$ by 0 on $\partial \Omega$.

Since $u$ is continuous on $\bar{\Omega}$, since $v$ is continuous when restricted to $\Omega \backslash U_{j}$ and since $u=v$ almost everywhere in $\Omega$ we have that $u=v$ pointwise on the open set $\Omega \backslash \bar{U}_{j}$ because all the points in this open set are Lebesgue points for both $u$ and $v$. We still have to show that $u=v$ on $\partial U_{j}$. Since the functions $u$ and $v$ agree on $\Omega \backslash \bar{U}_{j}$ and on $\partial \Omega$ and since they are both continuous when restricted to $\Omega \backslash U_{j}$, it follows that they agree on $\partial U_{j}$ as well. Therefore, $u=v$ on $\Omega \backslash U_{j}$ when $\Omega$ is bounded.

Case 2. We assume now that $\Omega$ is unbounded. We can assume without loss of generality that $0 \in \Omega$. Like in the proof of [7, Theorem 4.12], we choose a sequence of 2-Lipschitz smooth functions $\left(\phi_{m}\right)_{m \geq 1} \subset C_{0}^{\infty}\left(\mathbf{R}^{n}\right)$ such that $0 \leq \phi_{m} \leq 1, \phi_{m}=1$ on $B(0, m)$ and such that $\phi_{m}$ is compactly supported in $B(0, m+1)$ for every integer $m \geq 1$. For a fixed $m \geq 1$ let

$$
O_{k, m}=\left\{x \in \Omega:\left|\left(u_{k+1} \phi_{m}\right)(x)-\left(u_{k} \phi_{m}\right)(x)\right|>2^{-k}\right\} \text { and } U_{k, m}=\bigcup_{l \geq k} O_{l, m} .
$$


For every fixed $m \geq 1$ it is easy to see that $u \phi_{m} \in C(\Omega) \cap H_{0}^{1,(n, 1)}(\Omega)$ and that $\left(u_{k} \phi_{m}\right)_{k \geq 1} \subset C(\Omega) \cap H_{0}^{1,(n, 1)}(\Omega)$. Moreover,

$$
\begin{aligned}
\left\|u_{k} \phi_{m}-u \phi_{m}\right\|_{L^{(n, q)}(\Omega)} & \leq\left\|u_{k}-u\right\|_{L^{(n, q)}(\Omega)} \quad \text { and } \\
\left\|\nabla\left(u_{k} \phi_{m}\right)-\nabla\left(u \phi_{m}\right)\right\|_{L^{(n, q)}\left(\Omega ; \mathbf{R}^{n}\right)} & \leq\left\|\nabla u_{k}-\nabla u\right\|_{L^{(n, q)}\left(\Omega ; \mathbf{R}^{n}\right)}+2\left\|u_{k}-u\right\|_{L^{(n, q)}(\Omega)}
\end{aligned}
$$

for every $k \geq 1$. From our choice of the sequence of the sequence $\left(\phi_{m}\right)_{m \geq 1}$ it follows that

$O_{k} \cap B(0, m) \subset O_{k, m} \subset O_{k} \cap B(0, m+1)$ and $U_{k} \cap B(0, m) \subset U_{k, m} \subset U_{k} \cap B(0, m+1)$

for all integers $k, m \geq 1$.

By applying Case 1 to the sequence $\left(u_{k} \phi_{m}\right)_{k \geq 1}$ and to the bounded sets $U_{j, m} \subset$ $U_{j} \cap B(0, m+1)$ and $\Omega \cap B(0, m+1)$, we see that $u_{k} \phi_{m} \rightarrow u \phi_{m}$ uniformly on $\Omega \backslash U_{j, m}$ for every $m \geq 1$. From this, the definition of the functions $\phi_{m}$ and the fact that $u_{k} \rightarrow v$ uniformly on $\Omega \backslash U_{j}$, it follows that $u \phi_{m}=v \phi_{m}$ on $\Omega \backslash U_{j}$ for every $m \geq 1$. Thus, $u=v$ on $\Omega \backslash U_{j}$ when $\Omega$ is unbounded. This finishes the proof of Case 2 and the proof of the proposition.

\section{Choquet property for the capacities associated to $H_{0}^{1,(p, 1)}(\Omega)$}

In this section we prove that the Sobolev-Lorentz relative and global capacities defined via the $(p, 1)$ norm and respectively via the $p, 1$ norm have the Choquet property whenever $1 \leq n<p<\infty$ or $1<n=p<\infty$. We prove that all these set functions satisfy a Monotone Convergence Theorem type result whenever $1 \leq n<p<\infty$ or $1<n=p<\infty$. See Theorems 5.1 (v), 5.5 (v), 5.9 (iv) and respectively 5.12 (iv) and the discussions before and after Questions 5.3, 5.7, 5.11 and respectively 5.14 .

We start by showing that the Monotone Convergence Theorem holds for the $(p, 1)$ and the $p, 1$ relative capacities whenever $1 \leq n<p<\infty$ or $1<n=p<\infty$.

Theorem 8.1. Let $n \geq 1$ be an integer. Suppose that $1 \leq n<p<\infty$ or $1<n=p<\infty$. Let $\Omega \subset \mathbf{R}^{n}$ be bounded and open. Let $E_{k}$ be an increasing set of subsets in $\Omega$ and let $E=\bigcup_{k=1}^{\infty} E_{k}$. Then

(i) $\lim _{k \rightarrow \infty} \operatorname{cap}_{(p, 1)}\left(E_{k}, \Omega\right)=\operatorname{cap}_{(p, 1)}(E, \Omega)$,

(ii) $\lim _{k \rightarrow \infty} \operatorname{cap}_{p, 1}\left(E_{k}, \Omega\right)=\operatorname{cap}_{p, 1}(E, \Omega)$.

Proof. We start by proving claim (i). Due to the monotonicity of $\operatorname{cap}_{(p, 1)}(\cdot, \Omega)$, we have obviously

$$
L:=\lim _{k \rightarrow \infty} \operatorname{cap}_{(p, 1)}\left(E_{k}, \Omega\right)^{1 / p} \leq \operatorname{cap}_{(p, 1)}(E, \Omega)^{1 / p} .
$$

To prove the opposite inequality, we may assume without loss of generality that $L<\infty$. Let $\varepsilon \in(0,1)$ be fixed. For every $k \geq 1$ we choose $u_{k} \in \mathcal{A}\left(E_{k}, \Omega\right)$ such that $0 \leq u_{k} \leq 1$ and

$$
\left\|\nabla u_{k}\right\|_{L^{(p, 1)}\left(\Omega ; \mathbf{R}^{n}\right)}<\operatorname{cap}_{(p, 1)}\left(E_{k}, \Omega\right)^{1 / p}+\varepsilon
$$

for every $k \geq 1$.

Via Theorem 6.4 when $1<n=p<\infty$, via [7, Theorem 5.5 (iii)] when $1=n<$ $p<\infty$ or via [7, Theorem 5.6 (iv)] when $1<n<p<\infty$ we can assume without loss of generality ( since $u_{k}=1$ on an open neighborhood of $E_{k}$ ) that $u_{k}$ is in $C(\bar{\Omega}) \cap$ $H_{0}^{1,(p, 1)}(\Omega)$ and zero on $\partial \Omega$ for every $k \geq 1$. We notice that the sequence $\left(u_{k}\right)_{k \geq 1} \subset$ 
$C(\bar{\Omega}) \cap H_{0}^{1,(p, 1)}(\Omega)$ is bounded in $H_{0}^{1,(p, 1)}(\Omega)$ because the sequence $\left(u_{k}, \nabla u_{k}\right)_{k \geq 1}$ is bounded in $L^{(p, 1)}(\Omega) \times L^{(p, 1)}\left(\Omega ; \mathbf{R}^{n}\right)$.

Since $H_{0}^{1,(p, 1)}(\Omega) \subset H_{0}^{1, p}(\Omega)$ and the sequence $u_{k}$ is bounded in $H_{0}^{1,(p, 1)}(\Omega)$, it follows that $u_{k}$ is bounded in the reflexive space $H_{0}^{1, p}(\Omega)$. (See the discussion at the beginning of the proof of Theorem 7.1). Thus, via Theorem 4.4 there exists $u \in H_{0}^{1, p}(\Omega)$ and a subsequence, which we denote again by $u_{k}$, such that $\left(u_{k}, \nabla u_{k}\right) \rightarrow$ $(u, \nabla u)$ weakly in $L^{p}(\Omega) \times L^{p}\left(\Omega ; \mathbf{R}^{n}\right)$ as $k \rightarrow \infty$.

From Theorem 7.1 (ii) we can assume that $u$ is in fact in $C(\bar{\Omega}) \cap H_{0}^{1,(p, 1)}(\Omega)$ and $u=0$ on $\partial \Omega$. These assumptions can be made via Theorem 6.4 when $1<n=p<\infty$, via [7, Theorem 5.5 (iii)] when $1=n<p<\infty$ or via [7, Theorem 5.6 (iv)] when $1<n<p<\infty$. Moreover, from (9) and (28) we also have

$$
\|\nabla u\|_{L^{(p, 1)}\left(\Omega ; \mathbf{R}^{n}\right)} \leq \liminf _{k \rightarrow \infty}\left\|\nabla u_{k}\right\|_{L^{(p, 1)}\left(\Omega ; \mathbf{R}^{n}\right)} \leq L+\varepsilon .
$$

We want to show that $u=1$ on $E$. Let $v_{k, 3}$ be the sequence constructed in the proof of Theorem 7.1 (i) by applying Mazur's Lemma with respect to the sequence $\left(u_{k}, \nabla u_{k}\right)$ and the space $L^{p}(\Omega) \times L^{p}\left(\Omega ; \mathbf{R}^{n}\right)$ in order to prove $(9)$. Since the sets of admissible functions are closed under finite convex combinations and $E_{k} \nearrow E$ as $k \rightarrow \infty$, we have that $v_{k, 3} \in \mathcal{A}\left(E_{k}, \Omega\right)$ for every $k \geq 1$. In particular, $v_{k, 3}=1$ on an open neighborhood of $E_{k}$ for every $k \geq 1$.

We assume first that $1 \leq n<p<\infty$. By inspecting the proof of Theorem 7.1 (ii) (the case $\Omega$ bounded), we see that the functions $v_{k, 3}$ converge uniformly to $u$ on $\bar{\Omega}$ if $1 \leq n<p<\infty$. Since $v_{j, 3}$ is 1 on $E_{k}$ whenever $j \geq k \geq 1$, since the functions $v_{k, 3}$ converge uniformly to $u$ on $\bar{\Omega}$ and since $E_{k} \nearrow E$ as $k \rightarrow \infty$, it follows that $u=1$ on $E$ when $1 \leq n<p<\infty$. Thus, we proved that $u=1$ on $E$ if $1 \leq n<p<\infty$.

Assume now that $1<n=p<\infty$. By inspecting the proof of Proposition 7.3, we see that there exists a Borel set $F \subset \Omega$ such that $\operatorname{Cap}_{n}(F)=0$ and such that the sequence $v_{k, 3}$ converges to $u$ pointwise on $\bar{\Omega} \backslash F$. Similarly to the notation from Proposition 7.3, $F \subset \Omega$ is defined as $F:=\cap_{k \geq 1} U_{k}$, where $U_{k}=\cup_{j \geq k} O_{j}$ and

$$
O_{k}=\left\{x \in \Omega:\left|v_{k+1,3}(x)-v_{k, 3}(x)\right|>2^{-k}\right\}
$$

for every $k \geq 1$.

We see that $O_{j} \cap E_{k}=\emptyset$ whenever $j \geq k \geq 1$ because $v_{j, 3}=1$ on $E_{j} \supset E_{k}$ whenever $j \geq k \geq 1$. Thus, $U_{k} \cap E_{k}=\emptyset$ for every $k \geq 1$, which implies $F \cap E=\emptyset$. Thus, $v_{k, 3}$ converges to $u$ pointwise on $\bar{\Omega} \backslash F \supset E$. Since $v_{j, 3}=1$ on $E_{k}$ whenever $j \geq k \geq 1$ and since $E_{k} \nearrow E$ as $k \rightarrow \infty$, the pointwise convergence of $v_{k, 3}$ to $u$ on $E$ implies that $u=1$ on $E$ when $1<n=p<\infty$. Thus, we proved that $u=1$ on $E$ if $1<n=p<\infty$.

So far we showed that $u \in C(\bar{\Omega}) \cap H_{0}^{1,(p, 1)}(\Omega), u=0$ on $\partial \Omega$ and $u=1$ on $E$. We notice that $\frac{u}{1-\varepsilon} \in \mathcal{A}(E, \Omega)$. Thus, we have

$$
\operatorname{cap}_{(p, 1)}(E, \Omega)^{1 / p} \leq \frac{1}{1-\varepsilon}\|\nabla u\|_{L^{(p, 1)}\left(\Omega ; \mathbf{R}^{n}\right)} \leq \frac{1}{1-\varepsilon}(L+\varepsilon)
$$

for every $\varepsilon \in(0,1)$. By letting $\varepsilon \rightarrow 0$, we obtain

$$
\operatorname{cap}_{(p, 1)}(E, \Omega)^{1 / p} \leq L=\lim _{k \rightarrow \infty} \operatorname{cap}_{(p, 1)}\left(E_{k}, \Omega\right)^{1 / p} \leq \operatorname{cap}_{(p, 1)}(E, \Omega)^{1 / p} .
$$

This finishes the proof of the claim (i), namely the case of the $(p, 1)$ relative capacity. The proof of claim (ii), namely the case of the $p, 1$ relative capacity follows by doing an argument very similar to the argument used in the proof of claim (i). This finishes the proof of the theorem. 
From Theorem 8.1 (i) and the discussion before Question 5.3 it follows that the set function $\operatorname{cap}_{(p, 1)}(\cdot, \Omega)$ satisfies properties (i), (iv) and (v) of Theorem 5.1 whenever $1 \leq n<p<\infty$ or $1<n=p<\infty$. Thus, $\operatorname{cap}_{(p, 1)}(\cdot, \Omega)$ is a Choquet capacity (relative to $\Omega$ ) whenever $1 \leq n<p<\infty$ or $1<n=p<\infty$. Like in Theorem 5.1, the set $\Omega$ is bounded and open in $\mathbf{R}^{n}$, where $n \geq 1$ is an integer. We may invoke an important capacitability theorem of Choquet and state the following result. See Doob [9, Appendix II].

Theorem 8.2. Let $\Omega$ be a bounded open set in $\mathbf{R}^{n}$, where $n \geq 1$ is an integer. Suppose that $1 \leq n<p<\infty$ or $1<n=p<\infty$. The set function $E \mapsto \operatorname{cap}_{(p, 1)}(E, \Omega)$, $E \subset \Omega$, is a Choquet capacity. In particular, all Borel subsets (in fact, all analytic) subsets $E$ of $\Omega$ are capacitable, i.e.

$$
\operatorname{cap}_{(p, 1)}(E, \Omega)=\sup \left\{\operatorname{cap}_{(p, 1)}(K, \Omega): K \subset E \text { compact }\right\} \text {. }
$$

Similarly, from Theorem 8.1 (ii) and the discussion before Question 5.7 it follows that the set function $\operatorname{cap}_{p, 1}(\cdot, \Omega)$ satisfies properties (i), (iv) and (v) of Theorem 5.5 whenever $1 \leq n<p<\infty$ or $1<n=p<\infty$. Thus, $\operatorname{cap}_{p, 1}(\cdot, \Omega)$ is a Choquet capacity (relative to $\Omega$ ) whenever $1 \leq n<p<\infty$ or $1<n=p<\infty$. Like in Theorem 5.5, the set $\Omega$ is bounded and open in $\mathbf{R}^{n}$, where $n \geq 1$ is an integer. We may invoke an important capacitability theorem of Choquet and state the following result. See Doob [9, Appendix II].

Theorem 8.3. Let $\Omega$ be a bounded open set in $\mathbf{R}^{n}$, where $n \geq 1$ is an integer. Suppose that $1 \leq n<p<\infty$ or $1<n=p<\infty$. The set function $E \mapsto \operatorname{cap}_{p, 1}(E, \Omega)$, $E \subset \Omega$, is a Choquet capacity. In particular, all Borel subsets (in fact, all analytic) subsets $E$ of $\Omega$ are capacitable, i.e.

$$
\operatorname{cap}_{p, 1}(E, \Omega)=\sup \left\{\operatorname{cap}_{p, 1}(K, \Omega): K \subset E \text { compact }\right\} \text {. }
$$

Now we prove that the Monotone Convergence Theorem holds for the $(p, 1)$ and the $p, 1$ global capacities whenever $1 \leq n<p<\infty$ or $1<n=p<\infty$.

Theorem 8.4. Let $n \geq 1$ be an integer. Suppose that $1 \leq n<p<\infty$ or $1<n=p<\infty$. Let $E_{k}$ be an increasing set of subsets in $\mathbf{R}^{n}$ and let $E=\bigcup_{k=1}^{\infty} E_{k}$. Then

(i) $\lim _{k \rightarrow \infty} \operatorname{Cap}_{(p, 1)}\left(E_{k}\right)=\operatorname{Cap}_{(p, 1)}(E)$,

(ii) $\lim _{k \rightarrow \infty} \operatorname{Cap}_{p, 1}\left(E_{k}\right)=\operatorname{Cap}_{p, 1}(E)$.

Proof. We prove the claim in the case of the global $(p, 1)$-capacity. Due to the monotonicity of $\operatorname{Cap}_{(p, 1)}(\cdot)$, we have obviously

$$
L:=\lim _{k \rightarrow \infty} \operatorname{Cap}_{(p, 1)}\left(E_{k}\right)^{1 / p} \leq \operatorname{Cap}_{(p, 1)}(E)^{1 / p} .
$$

To prove the opposite inequality, we may assume without loss of generality that $L<\infty$. Let $\varepsilon \in(0,1)$ be fixed. For every $k \geq 1$ we choose $u_{k} \in S\left(E_{k}\right)$ such that $0 \leq u_{k} \leq 1$ and

$$
\left\|u_{k}\right\|_{H^{1,(p, 1)}\left(\mathbf{R}^{n}\right)}<\operatorname{Cap}_{(p, 1)}\left(E_{k}\right)^{1 / p}+\varepsilon
$$

for every $k \geq 1$.

Via Theorem 6.4 when $1<n=p<\infty$, via [7, Theorem 5.5 (iii)] when $1=$ $n<p<\infty$ or via [7, Theorem 5.6 (iv)] when $1<n<p<\infty$ we can assume without loss of generality (since $u_{k}=1$ on an open neighborhood of $E_{k}$ ) that $u_{k}$ is in $C\left(\mathbf{R}^{n}\right) \cap H_{0}^{1,(p, 1)}\left(\mathbf{R}^{n}\right)$ for every $k \geq 1$. We notice that the sequence $\left(u_{k}\right)_{k \geq 1} \subset$ 
$C\left(\mathbf{R}^{n}\right) \cap H_{0}^{1,(p, 1)}\left(\mathbf{R}^{n}\right)$ is bounded in $H_{0}^{1,(p, 1)}\left(\mathbf{R}^{n}\right)$ because the sequence $\left(u_{k}, \nabla u_{k}\right)_{k \geq 1}$ is bounded in $L^{(p, 1)}\left(\mathbf{R}^{n}\right) \times L^{(p, 1)}\left(\mathbf{R}^{n} ; \mathbf{R}^{n}\right)$.

Since $H_{0}^{1,(p, 1)}\left(\mathbf{R}^{n}\right) \subset H_{0}^{1, p}\left(\mathbf{R}^{n}\right)$ and the sequence $u_{k}$ is bounded in $H_{0}^{1,(p, 1)}\left(\mathbf{R}^{n}\right)$, it follows that $u_{k}$ is bounded in the reflexive space $H_{0}^{1, p}\left(\mathbf{R}^{n}\right)$. (See the discussion at the beginning of the proof of Theorem 7.1). Thus, via Theorem 4.4 there exists $u \in H_{0}^{1, p}\left(\mathbf{R}^{n}\right)$ and a subsequence, which we denote again by $u_{k}$, such that $\left(u_{k}, \nabla u_{k}\right) \rightarrow$ $(u, \nabla u)$ weakly in $L^{p}\left(\mathbf{R}^{n}\right) \times L^{p}\left(\mathbf{R}^{n} ; \mathbf{R}^{n}\right)$ as $k \rightarrow \infty$.

From Theorem 7.1 (ii) we can assume that $u$ is in fact in $C\left(\mathbf{R}^{n}\right) \cap H_{0}^{1,(p, 1)}\left(\mathbf{R}^{n}\right)$. These assumptions can be made via Theorem 6.4 when $1<n=p<\infty$, via [7, Theorem 5.5 (iii)] when $1=n<p<\infty$ or via [7, Theorem 5.6 (iv)] when $1<n<$ $p<\infty$. Moreover, from (7) and (29) we also have

$$
\|u\|_{H^{1,(p, 1)}\left(\mathbf{R}^{n}\right)} \leq \liminf _{k \rightarrow \infty}\left\|u_{k}\right\|_{H^{1,(p, 1)}\left(\mathbf{R}^{n}\right)} \leq L+\varepsilon .
$$

We want to show that $u=1$ on $E$. Let $v_{k, 1}$ be the sequence constructed in the proof of Theorem 7.1 (i) by applying Mazur's Lemma with respect to the sequence $\left(u_{k}, \nabla u_{k}\right)$ and the space $L^{p}\left(\mathbf{R}^{n}\right) \times L^{p}\left(\mathbf{R}^{n} ; \mathbf{R}^{n}\right)$ in order to prove (7). Since the sets of admissible functions are closed under finite convex combinations and $E_{k} \nearrow E$ as $k \rightarrow \infty$, we have that $v_{k, 1} \in S\left(E_{k}\right)$ for every $k \geq 1$. In particular, $v_{k, 1}=1$ on an open neighborhood of $E_{k}$ for every $k \geq 1$.

We assume first that $1 \leq n<p<\infty$. By inspecting the proof of Theorem 7.1 (ii) (the case $\Omega=\mathbf{R}^{n}$ ), we see that the functions $v_{k, 1}$ converge uniformly to $u$ on compact subsets of $\mathbf{R}^{n}$ if $1 \leq n<p<\infty$. Since $v_{j, 1}$ is 1 on $E_{k}$ whenever $j \geq k \geq 1$, since the functions $v_{k, 1}$ converge uniformly to $u$ on compact subsets of $\mathbf{R}^{n}$ and since $E_{k} \nearrow E$ as $k \rightarrow \infty$, it follows that $u=1$ on $E$ when $1 \leq n<p<\infty$. Thus, we proved that $u=1$ on $E$ if $1 \leq n<p<\infty$.

Assume now that $1<n=p<\infty$. By inspecting the proof of Proposition 7.3, we see that there exists a Borel set $F \subset \mathbf{R}^{n}$ such that $\operatorname{Cap}_{n}(F)=0$ and such that the sequence $v_{k, 1}$ converge to $u$ pointwise on $\mathbf{R}^{n} \backslash F$. Similarly to the notation from Proposition 7.3, $F \subset \mathbf{R}^{n}$ is defined as $F:=\bigcap_{k \geq 1} U_{k}$, where $U_{k}=\bigcup_{j \geq k} O_{j}$ and $O_{k}=\left\{x \in \mathbf{R}^{n}:\left|v_{k+1,1}(x)-v_{k, 1}(x)\right|>2^{-k}\right\}$.

We see that $O_{j} \cap E_{k}=\emptyset$ whenever $j \geq k \geq 1$ because $v_{j, 1}=1$ on $E_{j} \supset E_{k}$ whenever $j \geq k \geq 1$. Thus, $U_{k} \cap E_{k}=\emptyset$ for every $k \geq 1$, which implies $F \cap E=\emptyset$. Thus, $v_{k, 1}$ converges to $u$ pointwise on $\mathbf{R}^{n} \backslash F \supset E$. Since $v_{j, 1}=1$ on $E_{k}$ whenever $j \geq k \geq 1$ and since $E_{k} \nearrow E$ as $k \rightarrow \infty$, the pointwise convergence of $v_{k, 1}$ to $u$ on $E$ implies that $u=1$ on $E$ when $1<n=p<\infty$. Thus, we proved that $u=1$ on $E$ if $1<n=p<\infty$.

So far we showed that $u \in C\left(\mathbf{R}^{n}\right) \cap H_{0}^{1,(p, 1)}\left(\mathbf{R}^{n}\right)$ and $u=1$ on $E$. We notice that $\frac{u}{1-\varepsilon} \in S(E)$. Thus, we have

$$
\operatorname{Cap}_{(p, 1)}(E)^{1 / p} \leq \frac{1}{1-\varepsilon}\|u\|_{H^{1,(p, 1)}(\Omega)} \leq \frac{1}{1-\varepsilon}(L+\varepsilon)
$$

for every $\varepsilon \in(0,1)$. By letting $\varepsilon \rightarrow 0$, we obtain

$$
\operatorname{Cap}_{(p, 1)}(E)^{1 / p} \leq L=\lim _{k \rightarrow \infty} \operatorname{Cap}_{(p, 1)}\left(E_{k}\right)^{1 / p} \leq \operatorname{Cap}_{(p, 1)}(E)^{1 / p} .
$$

This finishes the proof of claim (i), namely the case of the $(p, 1)$ global capacity. The proof of claim (ii), namely the case of the global $p$,1-capacity follows by doing an argument very similar to the argument used in the proof of claim (i). This finishes the proof of the theorem. 
From Theorem 8.4 (i) and the discussion before Question 5.11 it follows that the set function $\operatorname{Cap}_{(p, 1)}(\cdot)$ satisfies properties (i), (iii) and (iv) of Theorem 5.9 whenever $1 \leq n<p<\infty$ or $1<n=p<\infty$. Thus, $\operatorname{Cap}_{(p, 1)}(\cdot)$ is a Choquet capacity when $1 \leq n<p<\infty$ or when $1<n=p<\infty$. Like in Theorem 5.9, $n \geq 1$ is an integer. We may invoke an important capacitability theorem of Choquet and state the following result. See Doob [9, Appendix II].

Theorem 8.5. Let $n \geq 1$ be an integer. Suppose that $1 \leq n<p<\infty$ or $1<n=p<\infty$. The set function $E \mapsto \operatorname{cap}_{(p, 1)}(E), E \subset \mathbf{R}^{n}$, is a Choquet capacity. In particular, all Borel subsets (in fact, all analytic) subsets $E$ of $\mathbf{R}^{n}$ are capacitable, i.e.

$$
\operatorname{Cap}_{(p, 1)}(E)=\sup \left\{\operatorname{Cap}_{(p, 1)}(K): K \subset E \text { compact }\right\} .
$$

Similarly, from Theorem 8.4 (ii) and the discussion before Question 5.14 it follows that the set function $\operatorname{Cap}_{p, 1}(\cdot)$ satisfies properties (i), (iii) and (iv) of Theorem 5.12 whenever $1 \leq n<p<\infty$ or $1<n=p<\infty$. Thus, $\operatorname{Cap}_{p, 1}(\cdot)$ is a Choquet capacity

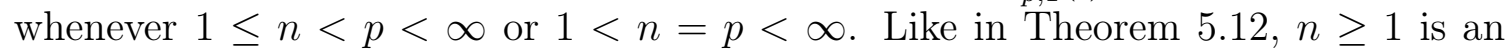
integer. We may invoke an important capacitability theorem of Choquet and state the following result. See Doob [9, Appendix II].

Theorem 8.6. Let $n \geq 1$ be an integer. Suppose that $1 \leq n<p<\infty$ or $1<n=p<\infty$. The set function $E \mapsto \operatorname{Cap}_{p, 1}(E), E \subset \mathbf{R}^{n}$, is a Choquet capacity. In particular, all Borel subsets (in fact, all analytic) subsets $E$ of $\mathbf{R}^{n}$ are capacitable, i.e.

$$
\operatorname{Cap}_{p, 1}(E)=\sup \left\{\operatorname{cap}_{p, 1}(K): K \subset E \text { compact }\right\} .
$$

Acknowledgements. I started writing this article towards the end of my stay at the University of Pisa and I finished it after my return to the University of Piteşti.

\section{References}

[1] Bennett, C., and R. Sharpley: Interpolation of operators. - Academic Press, 1988.

[2] Cianchi, A., and L. Pick: Sobolev embeddings into BMO, VMO, and $L_{\infty}$. - Ark. Mat. 36, $1998,317-340$.

[3] Cianchi, A., and L. Pick: Sobolev embeddings into spaces of Campanato, Morrey, and Hölder type. - J. Math. Anal. Appl. 282, 2003, 128-150.

[4] Costea, Ş.: Strong $A_{\infty}$-weights and scaling invariant Besov and Sobolev-Lorentz capacities. - Ph.D. thesis, University of Michigan, 2006.

[5] Costea, Ş.: Scaling invariant Sobolev-Lorentz capacity on $\mathbf{R}^{n}$. - Indiana Univ. Math. J. 56:6, 2007, 2641-2669.

[6] Costea, Ş.: Scaling Besov and Sobolev-Lorentz capacities in the Euclidean setting. - Publishing House of the Romanian Academy, Bucharest, 2012.

[7] CosteA, Ş.: Sobolev-Lorentz spaces in the Euclidean setting and counterexamples. - Nonlinear Anal. 152, 2017, 149-182.

[8] CosteA, Ş., and V. MAZ'YA: Conductor inequalities and criteria for Sobolev-Lorentz twoweight inequalities. - In: Sobolev Spaces in Mathematics II, Applications in Analysis and Partial Differential Equations, Springer, 2009, 103-121.

[9] Dоов, J. L.: Classical potential theory and its probabilistic cfounterpart. - Springer-Verlag, 1984.

[10] Evans, L. C.: Partial differential equations. - Amer. Math. Soc., 1998.

[11] Gilbarg, D., and N. S. Trudinger: Elliptic partial differential equations of second order. Springer-Verlag, 2nd edition, 1983. 
[12] Heinonen, J., T. Kilpeläinen, and O. Martio: Nonlinear potential theory of degenerate elliptic equations. - Oxford Univ. Press, 1993.

[13] Hunt, R.: On $L(p, q)$ measure spaces. - Enseign. Math. 12:2, 1966, 249-276

[14] Kauhanen, J., P. Koskela, and J. MalÝ: On functions with derivatives in a Lorentz space. - Manuscripta Math. 100:1, 1999, 87-101.

[15] Malý, J., D. Swanson, and W. P. Ziemer: Fine behavior of functions whose gradients are in an Orlicz space. - Studia Math. 190:1, 2009, 33-71.

[16] MaZ'YA, V.: Sobolev spaces. - Springer-Verlag, 1985.

[17] Stein, E.: Editor's note: The differentiability of functions in $\mathbf{R}^{n}$. - Ann. of Math. (2) 113, 1981, 383-385.

[18] Stein, E., and G. Weiss: Introduction to Fourier analysis on Euclidean spaces. - Princeton Univ. Press, 1975.

[19] Ziemer, W. P.: Weakly differentiable functions. - Springer-Verlag, 1989.

Received 20 February 2018 • Accepted 29 November 2018 